\title{
USP9X-mediated KDM4C deubiquitination promotes lung cancer radioresistance by epigenetically inducing TGF- $\beta 2$ transcription
}

\author{
Xiaohua Jie ${ }^{1}$ William Pat Fong ${ }^{1} \cdot$ Rui Zhou $^{1} \cdot$ Ye Zhao $^{1} \cdot$ Yingchao Zhao $^{1} \cdot$ Rui Meng $^{1} \cdot$ Sheng Zhang $^{1} \cdot$ \\ Xiaorong Dong ${ }^{1} \cdot$ Tao Zhang ${ }^{1} \cdot$ Kunyu Yang ${ }^{1} \cdot$ Gang Wu $\mathbb{D}^{1} \cdot$ Shuangbing Xu $\mathbb{1}^{1}$
}

Received: 27 September 2020 / Revised: 14 January 2021 / Accepted: 19 January 2021 / Published online: 8 February 2021

(c) The Author(s) 2021. This article is published with open access

\begin{abstract}
Radioresistance is regarded as the main barrier to effective radiotherapy in lung cancer. However, the underlying mechanisms of radioresistance remain elusive. Here, we show that lysine-specific demethylase 4C (KDM4C) is overexpressed and correlated with poor prognosis in lung cancer patients. We provide evidence that genetical or pharmacological inhibition of KDM4C impairs tumorigenesis and radioresistance in lung cancer in vitro and in vivo. Moreover, we uncover that KDM4C upregulates TGF- $\beta 2$ expression by directly reducing H3K9me3 level at the TGF- $\beta 2$ promoter and then activates Smad/ATM/Chk2 signaling to confer radioresistance in lung cancer. Using tandem affinity purification technology, we further identify deubiquitinase USP9X as a critical binding partner that deubiquitinates and stabilizes KDM4C. More importantly, depletion of USP9X impairs TGF- $\beta 2 /$ Smad signaling and radioresistance by destabilizing KDM4C in lung cancer cells. Thus, our findings demonstrate that USP9X-mediated KDM4C deubiquitination activates TGF- $\beta 2 /$ Smad signaling to promote radioresistance, suggesting that targeting KDM4C may be a promising radiosensitization strategy in the treatment of lung cancer.
\end{abstract}

\section{Introduction}

Cancer is as much a genetic disease as it is an epigenetic disease, and epigenetic alterations including histone modifications and DNA methylation are involved in tumor development and progression [1]. Histone lysine demethylase 4C (KDM4C, also known as JMJD2C/GASC1) is a member of the JmjC-domain-containing protein family

These authors contributed equally: Xiaohua Jie, William Pat Fong, Rui Zhou

Edited by H. Ichijo

Supplementary information The online version contains supplementary material available at https://doi.org/10.1038/s41418021-00740-z.

Gang Wu

xhzlwg@163.com

$\triangle$ Shuangbing Xu

xsb723@hust.edu.cn

1 Cancer Center, Union Hospital, Tongji Medical College, Huazhong University of Science and Technology, Wuhan 430022, China that encodes a histone demethylase for di- and trimethylated lysine 9 and 36 on histone 3 (H3K9me3/2 and H3K36me3/2) and has been shown to associate with malignant transformation [2,3]. Accumulating evidence suggests that $\mathrm{KDM} 4 \mathrm{C}$ is a candidate oncoprotein that is amplified or overexpressed in various cancer types [4-6]. In addition, several studies have demonstrated that KDM4C regulates the transcription of MYC, Il3ra and cyclin E1 by removing the repressive histone mark $\mathrm{H} 3 \mathrm{~K} 9 \mathrm{me} 3$, which in turn initiate metabolic reprogramming, control B cell activation and promote cell growth [7-10]. Excitingly, recent studies have demonstrated that SD70 is a novel KDM4C inhibitor and revealed its potential in impeding tumor progression in prostate cancer and acute myeloid leukemia [11, 12]. However, the regulatory mechanisms and roles of $\mathrm{KDM} 4 \mathrm{C}$ in cancer radioresistance have not yet been investigated.

Transforming growth factor- $\beta$ (TGF- $\beta$ ) is a critical modulator of numerous cellular activities that are central to tumorigenesis, with the main regulating pathways being canonical Smad-dependent and noncanonical Smadindependent signaling [13]. The TGF- $\beta /$ Smad signaling pathway has been pivotally implicated in many aspects of 
cellular processes, including tumor metastasis, immune regulation and microenvironment modification [14]. Importantly, the roles of TGF- $\beta /$ Smad signaling in the DNA damage response have also been documented [15-17]. For instance, Smad2 and Smad7 are critical intermediaries between TGF- $\beta$ and ataxia-telangiectasia mutated (ATM) crosstalk, while Smad3 binds to BRCA1 and inhibits BRCA1-dependent DNA damage repair $[15,16]$. In addition, preclinical studies using LY2109761, a novel TGF- $\beta$ receptor type I/II dual inhibitor, reported direct radiosensitizing effects in glioblastoma and breast cancer $[18,19]$. All these studies strongly support that activation of TGF- $\beta /$ Smad signaling is closely associated with cancer radioresistance.

In this study, we show that KDM4C is overproduced and predicts poor clinical outcomes in lung cancer patients. Furthermore, we present evidence that genetical or pharmacological inhibition of KDM4C overcomes radioresistance in lung cancer by inactivating the TGF- $\beta 2 / \mathrm{Smad} /$ ATM signaling pathway. Importantly, we are the first to report that the deubiquitinase USP9X is responsible for the upregulation of $\mathrm{KDM} 4 \mathrm{C}$ in lung cancer. These data reveal that $\mathrm{KDM} 4 \mathrm{C}$ is a promising therapeutic drug target and that its inhibitor SD70 is a potential radiosensitizer for lung cancer.

\section{Materials and methods}

\section{Cell culture and plasmid transfection}

All cell lines were purchased from American Type Culture Collection and cultured in either RPMI-1640 or DMEM supplemented with $10 \% \mathrm{FBS}$ and $100 \mathrm{IU} / \mathrm{mL}$ Penicillin-Streptomycin solution at $37^{\circ} \mathrm{C}$ in $5 \% \mathrm{CO}_{2}$. All cell lines were tested for mycoplasma contamination and authenticated using short tandem repeat (STR) profiling. Polymerase chain reaction (PCR) was used to generate the KDM4C plasmid, which was subcloned into the pDNOR201 entry vector and subsequently transferred to a gateway-compatible destination vector for the addition of SFB tag to express SFB-KDM4C protein using Gateway Technology (Invitrogen). Lipofectamine 2000 reagent (Invitrogen) was used for all plasmid transfections.

\section{Antibodies and reagents}

Several primary antibodies were used in this study: rabbit anti-KDM4A (Bethyl Laboratories, A300-861A, 1:1000), rabbit anti-KDM4B (Bethyl Laboratories, A301-478A, 1:1000), rabbit anti-KDM4C (Bethyl Laboratories, A300-885A, 1:800), rabbit anti-USP9X (Proteintech,
55054-1-AP, 1:1000), rabbit anti-HA (Cell Signaling Technology, \#3724, 1:1000), rabbit anti- $\beta$-Catenin (Cell Signaling Technology, \#8480, 1:1000), rabbit antiphospho-Chk2 (Thr68, Cell Signaling Technology, \#2197, 1:1000), rabbit anti-Smad2/3 (Cell Signaling Technology, \#8685, 1:1000), rabbit anti-phospho-Smad2 (Ser465/ Ser467, Cell Signaling Technology, \#18338, 1:1000), rabbit anti-phospho-Smad3 (Ser423/425, Cell Signaling Technology, \#9520, 1:1000), rabbit anti-TGF- $\beta 2$ (Proteintech, 19999-1-AP, 1:1000), rabbit anti-TGFßR1 (ABclonal, A16983, 1:1000), rabbit anti-TGFßR2 (ABclonal, A1415, 1:1000), mouse anti-GAPDH (ABclonal, AC033, 1:3000), rabbit anti-ATM (ABclonal, A19650, 1:1000), rabbit antiChk2 (ABclonal, A19543, 1:1000), mouse anti-Flag (Sigma-Aldrich, F1804, 1:1000), rabbit anti-H3K9me3 (Abcam, Ab8898, 1:800), rabbit anti-H3K36me3 (Abcam, Ab9050, 1:800), rabbit anti-H3 (Abcam, Ab1791, 1:1000), rabbit anti-phospho-ATM (Ser1981, Abcam, Ab81292, 1:1000) and rabbit anti-Rad51 (Abcam, Ab133534, 1:1000). The reagents used were as follows: SD70 (Xcess Biosciences, M60194-b), recombinant human TGF- $\beta 2$ (Abcam, ab84070), LY2109761 (Selleck, HY -12075), MG132 (Calbiochem, 474790) and cycloheximide (SigmaAldrich, A8244).

\section{RNA interference}

SiRNA transfection was carried out using Lipofectamine RNAiMAX transfection reagent (Invitrogen) for $48 \mathrm{~h}$. The following sequences of the siRNAs were used:

Scramble siRNA, 5'-UUCUCCGAACGUGUCACGU-3'; SiKDM4C\#1, 5'-AGAUAGCAGCAAUGAAGAA-3' [20]; SiUSP9X, 5'-AGAAAUCGCUGGUAUAAAUUU- $3^{\prime}$ [21-23];

SiTGF- $\beta 2$ \#1, 5'-GCAAUGGAGAAGAAUGCUU-3' [24]; SiTGF- $\beta 2$ \#2, 5'-GGAUUGAGCUAUAUCAGAU-3' [24].

\section{Establishment of stable lung cancer cell lines}

Stable KDM4C-depleted cell lines were established as previously described $[25,26]$. In brief, HEK293T cells were transfected with the indicated shRNAs, packaged plasmids psPAX2 and pMD2.G. After $48 \mathrm{~h}$, the supernatants were harvested and filtered using a $0.45 \mu \mathrm{m}$ filter. SPC-A1 and H460 cells were then infected using the filtered lentiviral supernatants with the addition of $10 \mu \mathrm{g} / \mathrm{mL}$ of polybrene (Sigma-Aldrich). Stable cells were screened using puromycin $(2 \mu \mathrm{g} / \mathrm{mL})$ and confirmed by Western blotting. The following sequences of the shRNAs were used:

Control shRNA: 5'-CTCGCTTGGGCGAGAGTAAG-3'; KDM4C shRNA-1: 5'-GCAGAGAGTAATGGTGTGT TA-3' [27];

KDM4C shRNA-2: 5'-TAGTGAATCGAACTTCTGG-3'. 
Tandem affinity purification S-Flag-SBP (SFB)tagged KDM4C complexes

This technology was conducted as previously described [26, 28, 29]. In brief, HEK293T cells stably expressing exogenous SFB-KDM4C were harvested and lysed with NETN buffer $(100 \mathrm{mM} \mathrm{NaCl}, 1 \mathrm{mM}$ EDTA, $20 \mathrm{mM}$ Tris$\mathrm{HCl}(\mathrm{pH} 8.0)$ and $0.5 \%$ Nonidet $\mathrm{P}-40$ ) at $4{ }^{\circ} \mathrm{C}$ for $20 \mathrm{~min}$. The lysates were centrifuged at $14000 \mathrm{rpm}$ and incubated with streptavidin-conjugated beads (Amersham) at $4{ }^{\circ} \mathrm{C}$ for $2 \mathrm{~h}$. Bead-bound proteins were washed and eluted twice using $2 \mathrm{mg} / \mathrm{mL}$ biotin (Sigma-Aldrich) diluted in NETN buffer for $2 \mathrm{~h}$. The elutes were further incubated with S-protein agarose (Novagen) for $1 \mathrm{~h}$. After being washed three times, the immunocomplexes were subjected to Western blotting. Protein bands were excised and further analyzed by mass spectrometry (performed by Taplin Mass Spectrometry Facility, Harvard Medical School).

\section{Western blotting and immunoprecipitation}

Cell lysates were prepared using NETN buffer and resolved by SDS-PAGE for Western blotting. The supernatants were initially incubated overnight with S-protein agarose (Novagen) at $4{ }^{\circ} \mathrm{C}$ for exogenous immunoprecipitation. For endogenous binding, cell lysates were incubated with the anti-KDM4C or USP9X antibody at $4{ }^{\circ} \mathrm{C}$ for $1 \mathrm{~h}$ before protein A/G agarose (Santa Cruz Biotechnology) was added. Lysates were then washed with NETN buffer five times prior to Western blotting analysis.

\section{In vivo ubiquitination assay}

Transfection of indicated siRNAs and corresponding plasmids into cells was carried out prior to the addition of $10 \mu \mathrm{M} \mathrm{MG132}$ for $4 \mathrm{~h}$ prior to harvesting. Cells were lysed using NETN buffer solution. S-protein agarose (Novagen) was then added and put on a rotating incubator overnight at $4{ }^{\circ} \mathrm{C}$. The beads were then washed five times, boiled with SDS loading buffer and subjected to Western blotting.

\section{RNA sequencing and analysis}

Total RNA was extracted from cells using TRIzol reagent (Invitrogen). RNA sequencing (RNA-seq) was performed by CapitalBio Technology (Beijing, China). RNA integrity was verified using an Agilent 2100 Bioanalyzer (Agilent Technologies). Paired-end library sequencing with Illumina HiSeq 2500 was performed with the mRNA-seq sample prep kit (Illumina) according to the manufacturer's specifications. The Illumina data analysis pipeline was then used for sequence analysis. All samples were allocated to lanes and processed in a blinded fashion to keep bias to a minimum.
The RNA-seq data have been deposited in the Gene Expression Omnibus database under accession number GSE136404 (https://www.ncbi.nlm.nih.gov/geo).

\section{Real-time quantitative PCR}

Extraction of total RNA from cell lysates was done using TRIzol reagent (Takara). cDNAs were synthesized using qPCR RT Master Mix (Toyobo, Osaka, Japan). Each PCR was performed in triplicate. The relative mRNA levels of target genes were determined by the $\Delta \mathrm{Ct}$ method (the $\mathrm{Ct}$ of GAPDH minus the $\mathrm{Ct}$ of the target gene). Primer sequences are given in Supplementary Table S1.

\section{Chromatin immunoprecipitation}

ChIP assay was performed following the protocol of the EZ$\mathrm{ChIP}^{\mathrm{TM}}$ Chromatin immune-precipitation kit (Millipore). The DNA pulled down by the anti-H3K9me3 or IgG antibody was amplified by PCR. The H3K9me3-bound DNA of TGF- $\beta 2$, BMP2 and JUND gene promoters was quantified by real-time PCR in triplicates using gene promoter-specific primers. Data were normalized to the corresponding DNA input control. The specific primers are listed in Supplementary Table S2.

\section{CCK-8 assay}

The half-maximal inhibitory concentrations (IC50s) of SD70 were analyzed using Cell count KIT-8 assay (CCK-8, Dojindo, Japan). Briefly, $1 \times 10^{3}$ cells were seeded in 96-well plates and cultured for $24 \mathrm{~h}, 48 \mathrm{~h}$ and $72 \mathrm{~h}$ respectively, followed by incubation with $8 \mu \mathrm{l} \mathrm{CCK-8}$ assay solution in each well for $1 \mathrm{~h}$. The absorbance values at $450 \mathrm{~nm}$ were measured using a Multimode Plate Reader (EnSpire 2300, USA).

\section{Cell clonogenic survival assay}

Equal number of SPC-A1 or H460 cells were plated in triplicate and grown in 6-well plates for each indicated irradiation (IR) dose. After exposure to IR, cells were cultured for $\sim 2$ weeks and washed with PBS buffer solution before fixation using methanol for $30 \mathrm{~min}$. Crystal violet staining solution $(0.5 \%)$ was used to dye cells. Colonies consisting of a nonoverlapping group of at least 50 cells were counted manually by a blinded reader using a light microscope. The single-hit multi-target model was used to calculate the surviving fraction, which was performed as previously described [30].

\section{Neutral comet assay}

The neutral comet assay was performed in triplicate using the Comet Assay Kit (Trevigen, 4250-050-K) according to 
the manufacturer's specifications. In brief, cells were irradiated at $6 \mathrm{~Gy}$ and collected after $4 \mathrm{~h}$, after which they were immobilized on the comet slide using low melting agarose. Lysis was performed for $1 \mathrm{~h}$ and washed three times with neutral electrophoresis buffer before being subjected to electrophoresis at $4{ }^{\circ} \mathrm{C}$ for $1 \mathrm{~h}$. Gels were then neutralized and stained using SYBR Gold (Invitrogen), and cells were viewed using a fluorescence microscope and analyzed using CometScore 2.0 and GraphPad Prism Software. Olive tail moments were analyzed using at least 100 cells from each group.

\section{Immunofluorescence staining}

SPC-A1 or H460 cells transfected with KDM4C targeting shRNAs or treated with SD70 were seeded on coverslips and subjected to 6 Gy IR. At $4 \mathrm{~h}$ postirradiation, cells were fixed using $4 \%$ paraformaldehyde, permeabilized with $0.2 \%$ Triton X-100 for 15 min and then blocked with 5\% bovine serum albumin. Subsequently, the samples were incubated with anti-phospho-ATM (Ser1981, 1:500), anti-phosphoChk2 (Thr68, 1:500) or anti-Rad51 (1:500) antibodies overnight and secondary antibody for $1 \mathrm{~h}$. Cells were counterstained with DAPI for $10 \mathrm{~min}$ and observation was done by fluorescence microscope.

\section{Animal experiments}

All animal experiments were approved by The Medical Ethics Committee of Tongji Medical College, Huazhong University of Science and Technology. Four- to six-weekold BALB/c nude mice were allocated randomly into four groups (eight mice per group) and were injected subcutaneously with $5 \times 10^{6}$ SPC-A1 stable cells (shControl, shKDM4C-1, shKDM4C-2). A radiation dose of 10 Gy was given when tumors reached a volume of $130 \mathrm{~mm}^{3}$.

For SD70 treatment, SD70 drug preparation and dosing for xenograft experiments were performed as described [11]. Briefly, nude BALB/c mice were allocated at random into four groups (eight mice per group), and lung tumor cells were implanted subcutaneously. SD70 was administered intraperitoneally at $10 \mathrm{mg} / \mathrm{kg}$ in PEG300/D5W starting from the first day when the tumor volume reached a calculated average of $130 \mathrm{~mm}^{3}$ and continued for 4 weeks with five consecutive injections in the first week and every other day for the next 3 weeks. Mice in the IR groups were subjected to $10 \mathrm{~Gy}$ local IR using an X-ray irradiator (Varian, USA) at a dose rate of 6 $\mathrm{Gy} / \mathrm{min}$ on the day after the first injection of SD70 or vehicle, which was previously described [30]. All tumors were measured by a blinded reader using Vernier calipers every 3 days, and the formula used to calculate tumor volumes was as follows: $\mathrm{V}=\left(\mathrm{L} \times \mathrm{W}^{2}\right) / 2$, where $\mathrm{V}=$ volume $\left(\mathrm{mm}^{3}\right), \mathrm{L}=$ length $(\mathrm{mm})$, and $\mathrm{W}=$ width $(\mathrm{mm})$.

\section{Lung cancer tissue microarray and immunohistochemical (IHC) staining}

Lung adenocarcinoma tissue microarrays containing lung carcinoma tissues and paired healthy lung tissues were provided by Outdo Biotech (Shanghai, China). IHC analysis was carried out as previously described $[28,29]$. The percentage of the positive area and the staining intensity were both used to evaluate KDM4C and USP9X expression. The staining index (values $0-12$ ) indicated the percentage of positively stained cells $(0-25 \%=1, \quad 26-50 \%=2, \quad 51-75 \%=3,>$ $75 \%=4$ ) and the intensity of positive staining (negative $=0$, weak $=1$, moderate $=2$, or strong $=3$ ) and was calculated by multiplying these two values; a score $<8$ was defined as low expression, and a score equal to or higher than eight was deemed high expression.

\section{Statistics analysis}

All in vitro experiments were carried out independently and in triplicate. All quantitative data are presented as the mean \pm SD unless stated otherwise. All statistical power analysis for group comparisons were performed using Student's $t$ test (two-tailed). The Kaplan-Meier method was used to evaluate overall survival. Pathway enrichment analysis was performed using the PANTHER pathway database. $P$ value $<0.05$ were considered statistically significant $(* P<0.05, * * P<0.01, * * * P<0.001)$.

\section{Results}

\section{KDM4C is overproduced and predicts poor clinical outcomes in lung cancer patients}

To evaluate the clinical significance of KDM4C in lung cancer, we first detected the protein level of KDM4C in six human lung cancer cell lines and as predicted, KDM4C protein level was upregulated compared to that in normal human lung epithelial cell HBE (Fig. 1a, b). Next, using immunohistochemistry (IHC) analysis, we quantified KDM4C protein levels in a human lung cancer tissue microarray consisting of 86 fresh lung adenocarcinoma tissues matched with their adjacent nontumor tissues (NTs) and demonstrated that KDM4C localized to the nucleus and cytoplasm of cancer cells and its protein levels were considerably higher in lung cancer tissues than in adjacent NTs (Fig. 1c, d). Moreover, we showed that there was a correlation between high expression of KDM4C and shorter overall survival in lung cancer patients by Kaplan-Meier survival analysis $(P<0.05)$ (Fig. 1e). These data suggest that $\mathrm{KDM} 4 \mathrm{C}$ is overexpressed and related to a worse prognosis in lung cancer. 
a

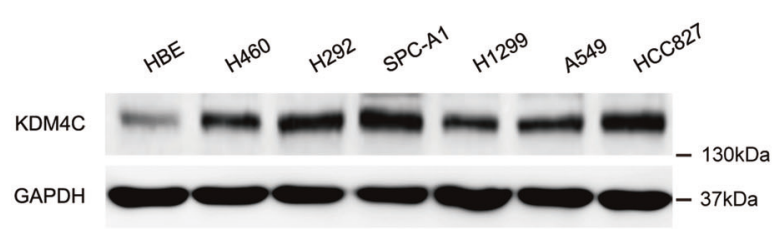

C

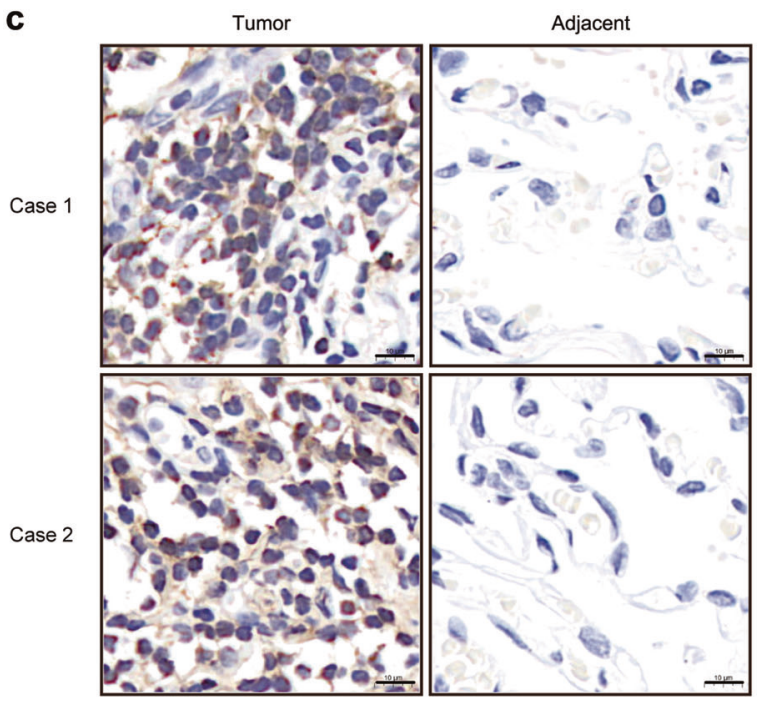

b

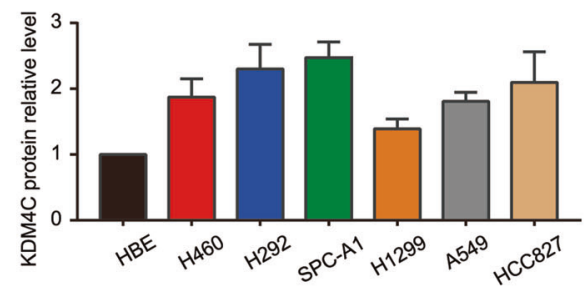

d

\begin{tabular}{cccc}
\hline & \multicolumn{2}{c}{ KDM4C Expression } & \multirow{2}{*}{ Total } \\
\cline { 2 - 3 } & High (\%) & Low (\%) & \\
\hline Tumor & $63(73.3)$ & $23(26.7)$ & 86 \\
Adjacent & $32(37.2)$ & $54(62.8)$ & 86 \\
\hline & & & $P<0.001$
\end{tabular}

e

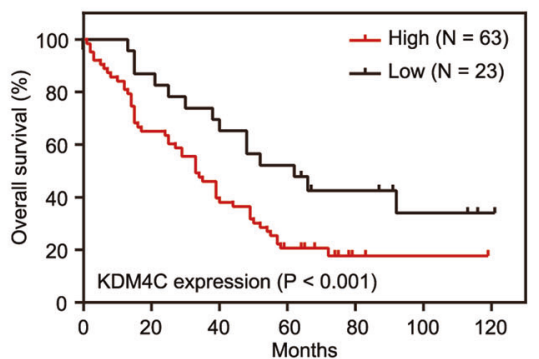

Fig. $1 \mathrm{KDM} 4 \mathrm{C}$ is upregulated and predicts poor clinical outcomes in lung cancer. a KDM4C protein levels in different cell lines were examined by Western blotting. b Quantification of KDM4C protein levels in (a) $(n=3)$. c Representative IHC staining pictures for $\mathrm{KDM} 4 \mathrm{C}$ in lung adenocarcinoma tissues and adjacent lung tissues.

\section{KDM4C silencing impairs tumorigenesis and enhances radiosensitivity in lung cancer in vitro and in vivo}

Considering that $\mathrm{KDM} 4 \mathrm{C}$ is substantially overexpressed in lung cancer tissues, we assumed that it might exert a tumorigenic effect in lung cancer. To this point, we first constructed KDM4C knockdown lung cancer cells using shRNAs (Fig. 2a) and found that loss of KDM4C inhibited cell proliferation (Fig. 2b). Subsequently, we inoculated Tcell-deficient athymic nude mice with KDM4C control and knockdown cells and showed that shKDM4C xenografts displayed significantly decreased tumor growth and tumor weight compared to control xenografts (Fig. 2c, d). These findings suggest that $\mathrm{KDM} 4 \mathrm{C}$ silencing impairs tumorigenesis in lung cancer in vitro and in vivo.

It has been reported that the specific substrates of KDM4C, $\mathrm{H} 3 \mathrm{~K} 9$, and $\mathrm{H} 3 \mathrm{~K} 36$ are closely linked with double-strand break repair [31, 32], therefore we speculated that KDM4C might be involved in regulating radiosensitivity. To test this hypothesis, we first performed a neutral comet assay and found that the olive tail moment is higher in KDM4C deficient cells compared to that in control cells (Fig. 2e).
Scale bar, $10 \mu \mathrm{m}$. d Statistical analysis of IHC staining to assess KDM4C expression in a lung cancer tissue microarray. e High KDM4C expression is correlated with adverse overall survival in lung cancer patients.

In agreement with this idea, DNA damage-induced Rad51 foci was decreased in KDM4C knockdown cells (Fig. 2f). These results imply that inhibiting KDM4C accelerates DNA damage and impedes DNA repair. To further validate our findings, we used a clonogenic survival assay to determine the growth ability of cells after IR. As expected, loss of KDM4C enhanced cellular sensitivity to radiation (Fig. $2 \mathrm{~g}$ ). Next, we examined the effect of KDM4C knockdown in xenografts exposed to IR. As shown in Fig. 2h, the post-irradiation control group had a smaller tumor size compared to the nonexposed control group, indicating that IR was effective. More importantly, the post-IR shKDM4C group showed an even more significant decrease in tumor size compared to the postIR control group (Fig. 2h). These results suggest that targeting KDM4C impedes tumorigenesis and renders lung cancer cells more susceptible to IR in vitro and in vivo.

\section{Inhibition of KDM4C with a pharmacologic drug SD70 hinders lung cancer progression and enhances response to radiotherapy in vitro and in vivo}

To further evaluate the therapeutic potential of KDM4C inhibition, we used SD70, a selective competitive inhibitor 


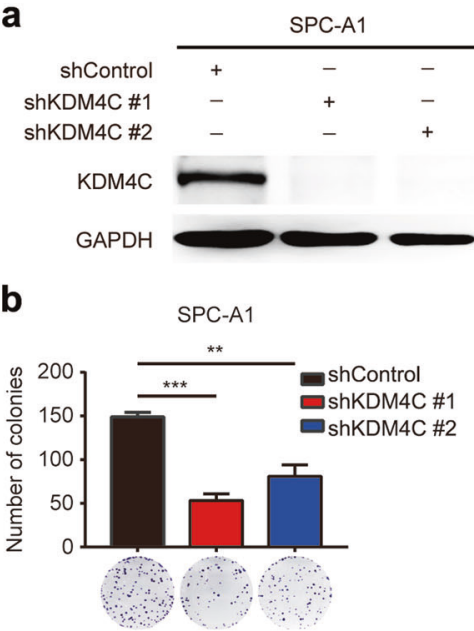

e
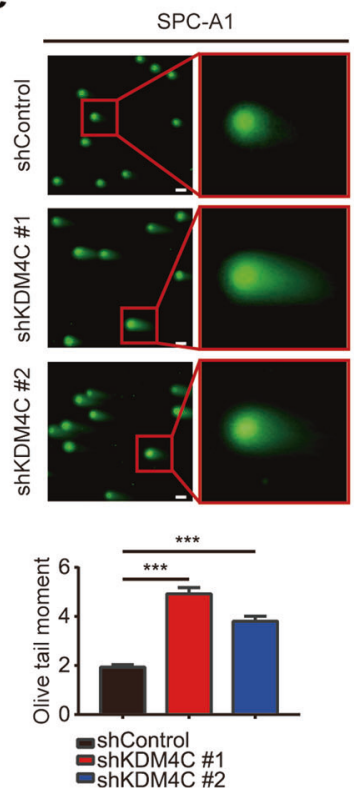

g

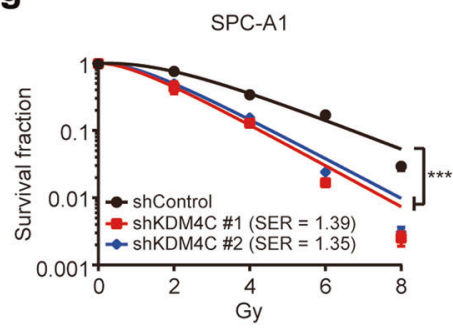

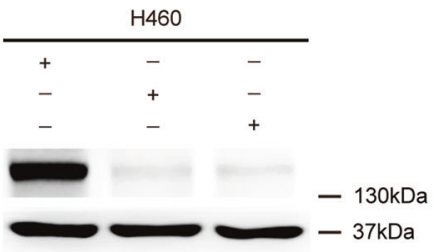

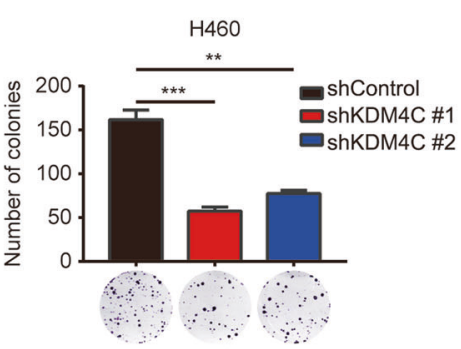

C

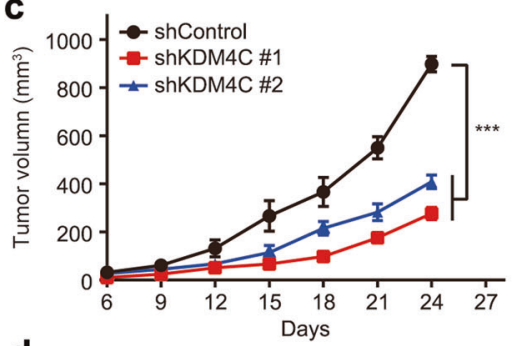

d

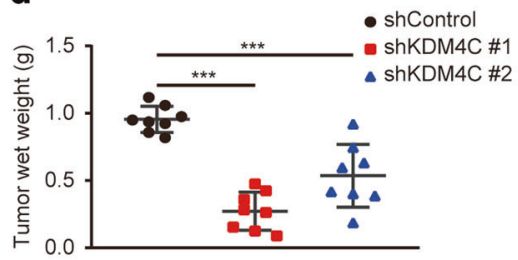

f
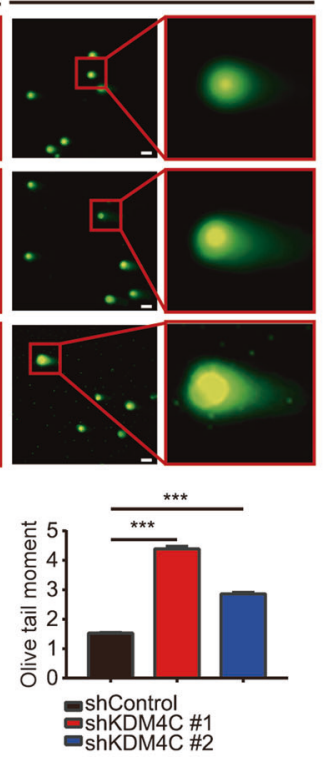

SPC-A1
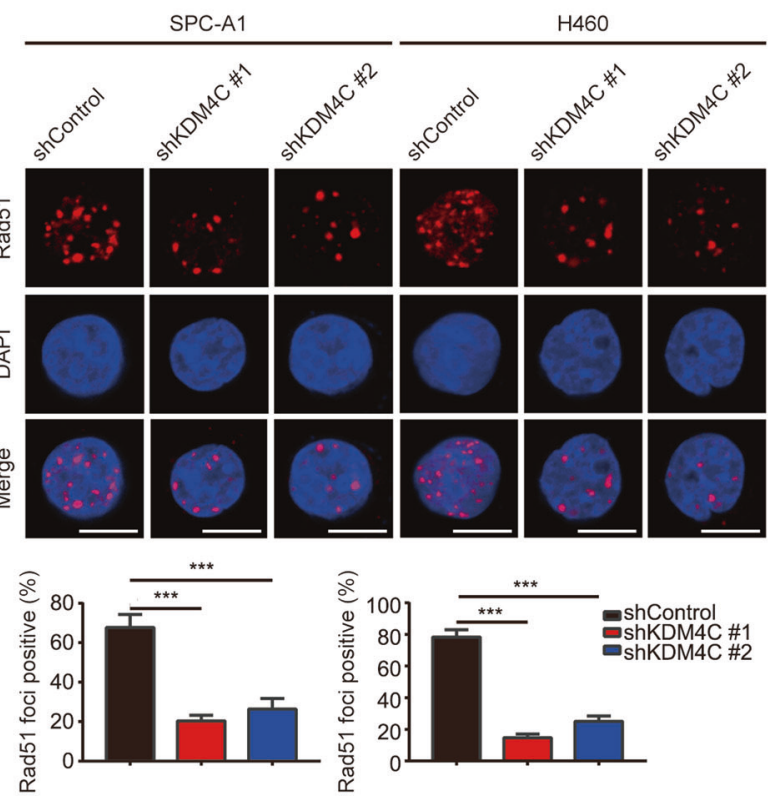

h

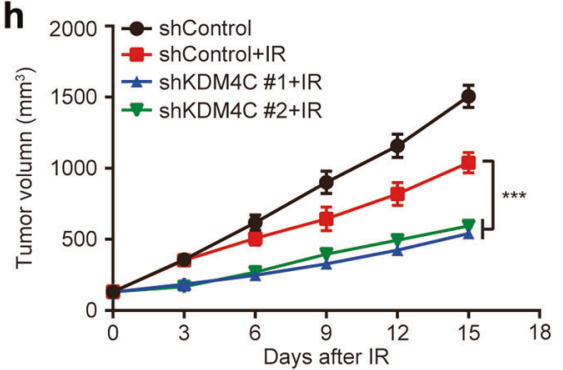

Fig. 2 KDM4C depletion suppresses tumorigenesis and enhances radiosensitivity in lung cancer in vitro and in vivo. a SPC-A1 and H460 cells were transfected with the control or shKDM4C lentivirus, and samples were collected and analyzed by Western blotting using indicated antibodies $(n=3)$. b SPC-A1 and H460 cells stably expressing control or KDM4C shRNAs were seeded and cultured for 2 weeks. Crystal violet was then used to stain colonies which were counted under a microscope. $* * P<0.01, * * * P<0.001(n=3)$. c $5 \times 10^{6}$ SPC-A1 stable cells (ShControl or Sh-KDM4C) were subcutaneously injected into BALB/c nude mice. The growth curves of the xenograft tumors in the three groups are presented ( $n=8$ mice for each group). $* * * P<0.001$. d Tumor weights in three groups were shown $(n=8$ mice for each group). $* * * P<0.001$. e Representative pictures of comet assays performed $4 \mathrm{~h}$ after IR exposure of KDM4C-depleted or control cells. $* * * P<0.001(n=3)$. Scale bar, 20 $\mu \mathrm{m}$. f KDM4C-depleted SPC-A1 and H460 cells were irradiated and harvested $4 \mathrm{~h}$ later. Upper panel: Representative immunostaining pictures of Rad51 foci. Lower panel: Rad51 foci quantification results. A cell containing 10 or more foci was considered as a foci-positive cell. $* * * P<$ $0.001(n=3)$. Scale bar, $10 \mu \mathrm{m}$. g KDM4C silencing led to enhanced radiosensitivity. Data are presented as the mean \pm SD. SER: sensitization enhancement ratio. $* * P<0.01, * * * P<0.001 \quad(n=3)$. h The growth curves of xenograft tumors for each group are represented. Data are presented as mean $\pm \operatorname{SEM}(n=8$ mice for each group). $* * * P<0.001$. 
of KMD4C, to block its catalytic domain without affecting its expression and tested its efficacy on lung cancer cell functions. Using CCK-8 assay, we determined the halfmaximal inhibitory concentrations (IC50s) of SD70 in SPCA1 (24 h) and H460 (48 h) cell lines, and we used $3 \mu \mathrm{M}$ SD70 for all subsequent experiments (Supplementary Fig. 1). As shown in Fig. $3 \mathrm{a}$ and b, the accumulation of both H3K9me3 and H3K36me3 was time and concentrationdependent when cells were treated with SD70, while KDM4C levels remained constant, validating that SD70 can indeed inhibit the activity of KDM4C. Furthermore, we found that SD70 was able to suppress lung cancer cell proliferation in vitro (Fig. 3c). Consistent with our in vitro results, KDM4C inhibition effectively hindered tumor growth in xenografts in a concentration-dependent manner (Fig. 3d). Importantly, the weight of nude mice remained constant, implying that SD70 is non-toxic and safe (Fig. 3e). In addition, we also evaluated the potential pharmacological effects of SD70 in radiosensitization. As shown in Fig. 3f and g, increased olive tail moment and impaired Rad51 foci formation were observed when cells were treated with SD70. In line with these notions, SD70 treatment enhanced cell radiosensitivity to IR (Fig. 3h). Next, we assessed the pharmacological efficacy of SD70 in vivo using the experimental workflow (Fig. 3i). The growth of tumor xenografts was slightly impaired when inoculated nude mice were treated with $10 \mathrm{mg} / \mathrm{kg}$ SD70 (Fig. 3j). However, when xenografts were exposed to a combination of SD70 (10 mg/kg) and IR, the tumor growth was considerably subdued compared to the group exposed to IR only (Fig. 3j). Moreover, mice from the combined treatment group had a strikingly better overall survival (Fig. 3k). These results provide preclinical evidence for SD70 as a conceivable therapeutic drug in the treatment of lung cancer, especially as a radiosensitizer.

\section{KDM4C induces the expression of TGF- $\beta 2$ and then activates Smad signaling by removing the repressive mark $\mathrm{H} 3 \mathrm{~K} 9 \mathrm{me} 3$ at the TGF- $\beta 2$ promoter}

To identify the molecular pathways by which KDM4C exerts its biological effects in lung cancer, we employed RNA sequencing (RNA-Seq) screening and identified the expression of $\sim 544$ genes with significant changes $(P$ value $<0.05$ and foldchange absolute value $\geq 2$ ) in KDM4C-depleted cells compared to that in control cells (Fig. 4a). Further pathway analysis demonstrated that the TGF- $\beta$ signaling was significantly altered (Fig. 4b), indicating KDM4C may activate the TGF- $\beta$ signaling pathway. RNA-seq results showed that a total of seven differentially expressed genes with more than twofold change were involved in the TGF- $\beta$ signaling pathway (Fig. 4c). Several candidate genes were further validated. As illustrated in Fig. 4d and e, inhibition of KDM4C with
shRNAs or SD70 treatment reduced the basal expression levels of TGF- $\beta 2$, BMP 2 and JUND while increasing the expression level of GDF11 in lung cancer cells. It has been well established that KDM4C-mediated reduction of H3K9me3 level often leads to transcriptional activation of target genes [2]. To determine whether KDM4C could directly regulate the $\mathrm{H} 3 \mathrm{~K} 9 \mathrm{me} 3$ levels at the promoter of TGF$\beta 2$, BMP2 and JUND, we performed ChIP-qPCR analysis and revealed that $\mathrm{KDM} 4 \mathrm{C}$ silencing caused substantial increases in the level of $\mathrm{H} 3 \mathrm{~K} 9 \mathrm{me} 3$ at the TGF- $\beta 2$ promoter, but not BMP2 and JUND promoters (Fig. 4f-h and Supplementary Fig. 2). These results indicate that KDM4C is needed for the transcription of TGF- $\beta 2$ in lung cancer cells.

Given that KDM4C positively controls the expression of TGF- $\beta 2$, we speculated that KDM4C may be a key activator of TGF- $\beta 2 /$ Smad signaling. To test this idea, we depleted KDM4C using shRNAs and found that TGF- $\beta 2$, phosphorylated Smad2 and Smad 3 levels (p-Smad2 and pSmad3) were decreased while TGF $\beta R 1$, TGF $\beta$ R2 and total Smad 2/3 remained constant when KDM4C was knocked down (Fig. 5a). Consistently, we observed similar results when KDM4C was inhibited using SD70 (Fig. 5b). In addition, it has been reported that TGF- $\beta$ is necessary for DNA double-strand breaks recognition and regulates the kinase activity of ATM $[16,17]$, we therefore surmised that KDM4C might be involved in the activation of ATM signaling. To that end, we detected molecular changes of the ATM pathway and uncovered that both phosphorylated ATM and Chk2 levels were decreased while total ATM and Chk2 remained constant in response to DNA damage when KDM4C was inhibited (Fig. 5a, b). In line with the above results, DNA damage induced phospho-ATM and phosphoChk2 foci formation were also impaired when KDM4C was blocked by shRNAs or SD70 (Fig. 5c-f). These data indicate that KDM4C stimulates TGF- $\beta 2 / \mathrm{Smad}$ signaling to activate the downstream ATM/Chk2 pathway in lung cancer.

\section{Tumor growth suppression and enhanced radiosensitivity caused by KDM4C silencing are dependent on the TGF- $\beta 2 /$ Smad signaling pathway inactivation}

To confirm the involvement of the TGF- $\beta 2 /$ Smad signaling pathway in KDM4C mediated effects in lung cancer, KDM4C and TGF- $\beta 2$ were silenced with siRNAs and recombinant human TGF- $\beta 2$ was added into KDM4C-depleted lung cancer cells. As shown in Fig. 6a, c, e and Supplementary Fig. 3, knockdown of TGF- $\beta 2$ reduced the expression levels of $p$ Smad 2 and p-Smad3 as well as suppressed lung cancer cell growth and proliferation whereas exogenous TGF- $\beta 2$ elicited the opposite effects, suggesting that TGF- $\beta 2$ activates Smad signaling and plays an oncogenic role in lung cancer. 
a

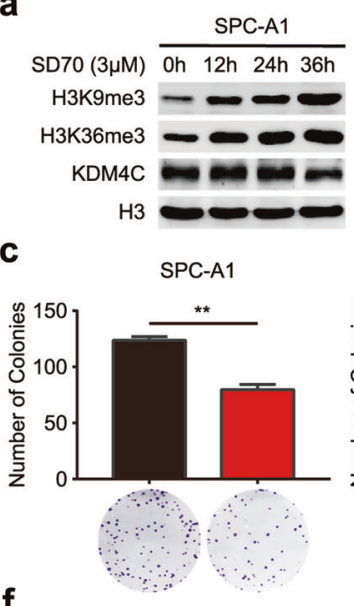

f
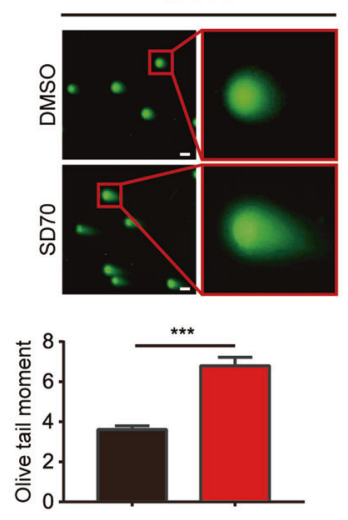

$\mathrm{H} 460$

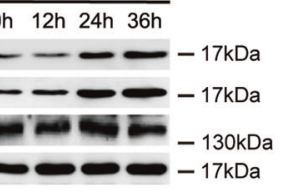

b

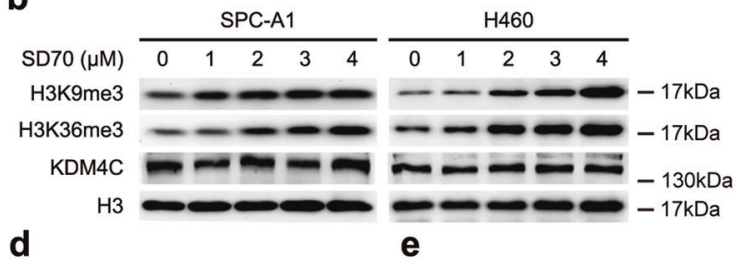

d
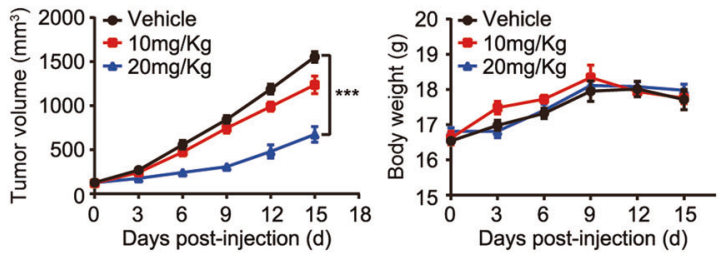

g
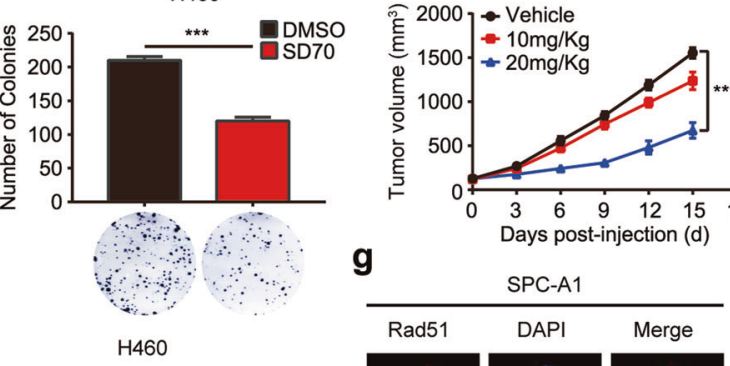

$\mathrm{H} 460$

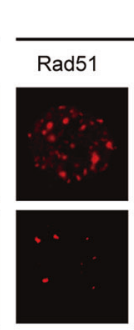

DAPI Merge
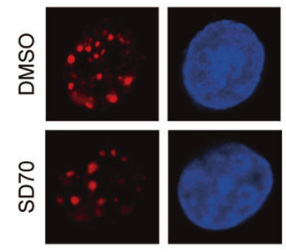

SPC-A1
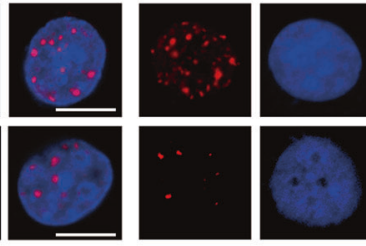

$\mathrm{H} 460$
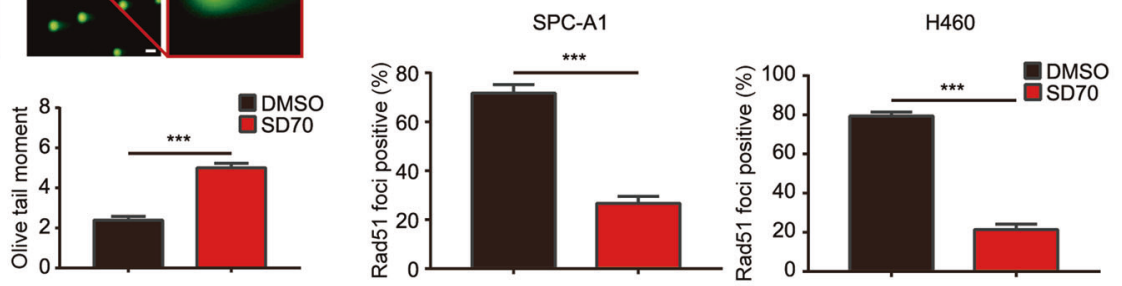

h

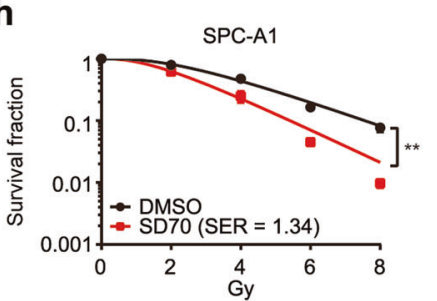

i

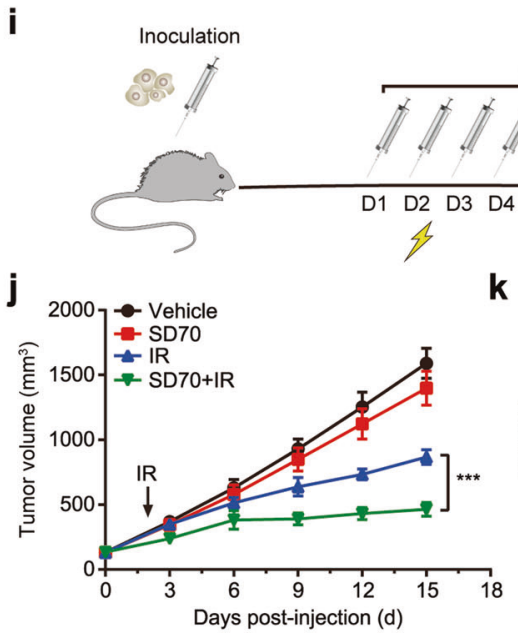

SD70/DMSO (i.p.)
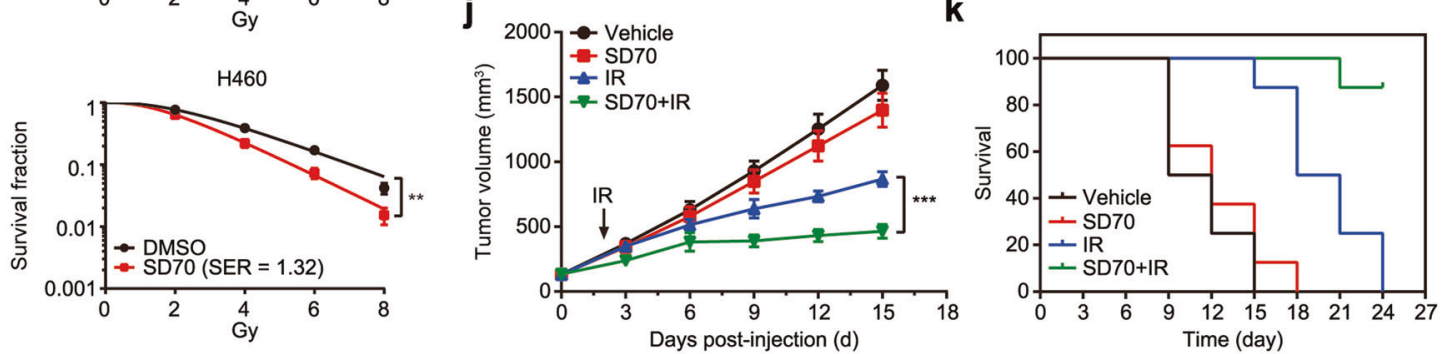

Fig. 3 Inhibition of KDM4C with SD70 impairs cell proliferation and radioresistance in lung cancer in vitro and in vivo. a, b The inhibition efficacy of SD70 is time and concentration-dependent $(n=3)$. c SPC-A1 and H460 cells were treated with SD70 $(3 \mu \mathrm{M})$ for $24 \mathrm{~h}$ and $48 \mathrm{~h}$, respectively. Cells were then seeded and cultured for 2 weeks. The colonies were stained using crystal violet solution and then counted. Representative pictures are shown. $* * P<0.01, * * * P<$ $0.001(n=3)$. d Inhibition of KDM4C using indicated doses of SD70 impairs tumor growth in lung cancer in vivo $(n=5$ mice for each group). $* * * P<0.001$. e Changes in body weight of xenografts postinoculation were represented. $\mathbf{f}$ Neutral comet assay shows the effect of SD70 treatment on tail formation. The olive tail moment was quantified and graphed. $* * * P<0.001(n=3)$. Scale bar, $20 \mu \mathrm{m}$. g Quantification of Rad51 foci intensity in SPC-A1 and H460 cells after treatment with SD70. Representative images are shown. Scale bar, $10 \mu \mathrm{m} .{ }^{* * *} P<0.001(n=3)$. h The effects of SD70 treatment on the radiosensitivity of lung cancer cells were determined by clonogenic survival assay. $* * P<0.01, * * * P<0.001(n=3)$. i Schematic representation of the xenograft study design and experimental workflow. j Growth curves of xenograft tumors in each group. Mice were treated with SD70 at the concentration of $10 \mathrm{mg} / \mathrm{kg}(n=8$ mice for each group). $* * * P<0.001$. k Kaplan-Meier analysis of mice survival due to treatments in $(\mathbf{j})$. 


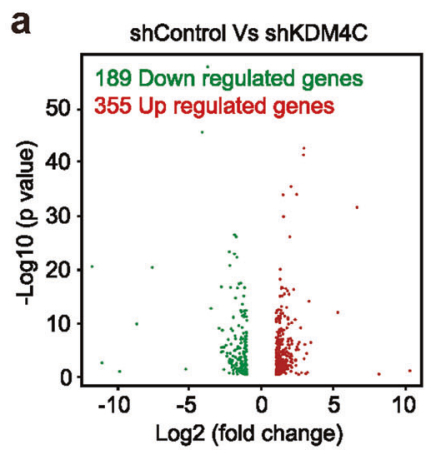

C

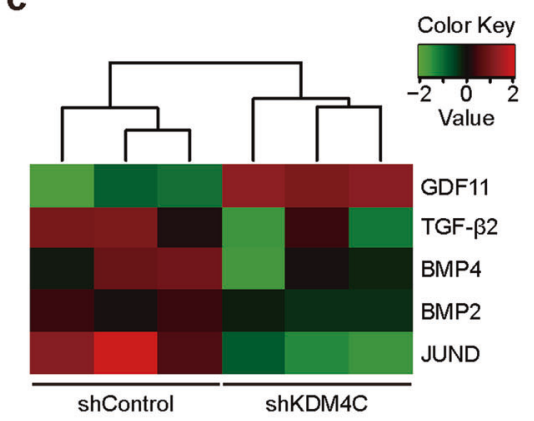

f

TGF- $\beta 2$

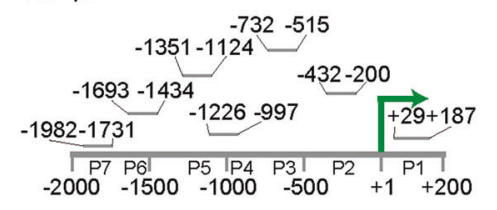

g

BMP2

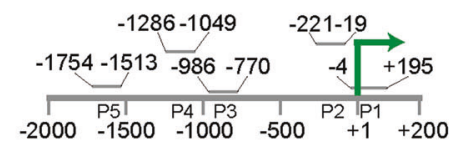

h

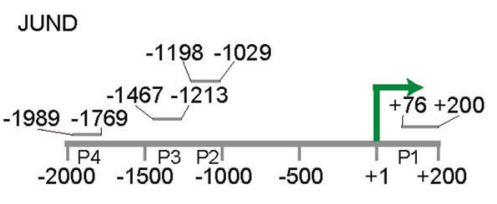

b
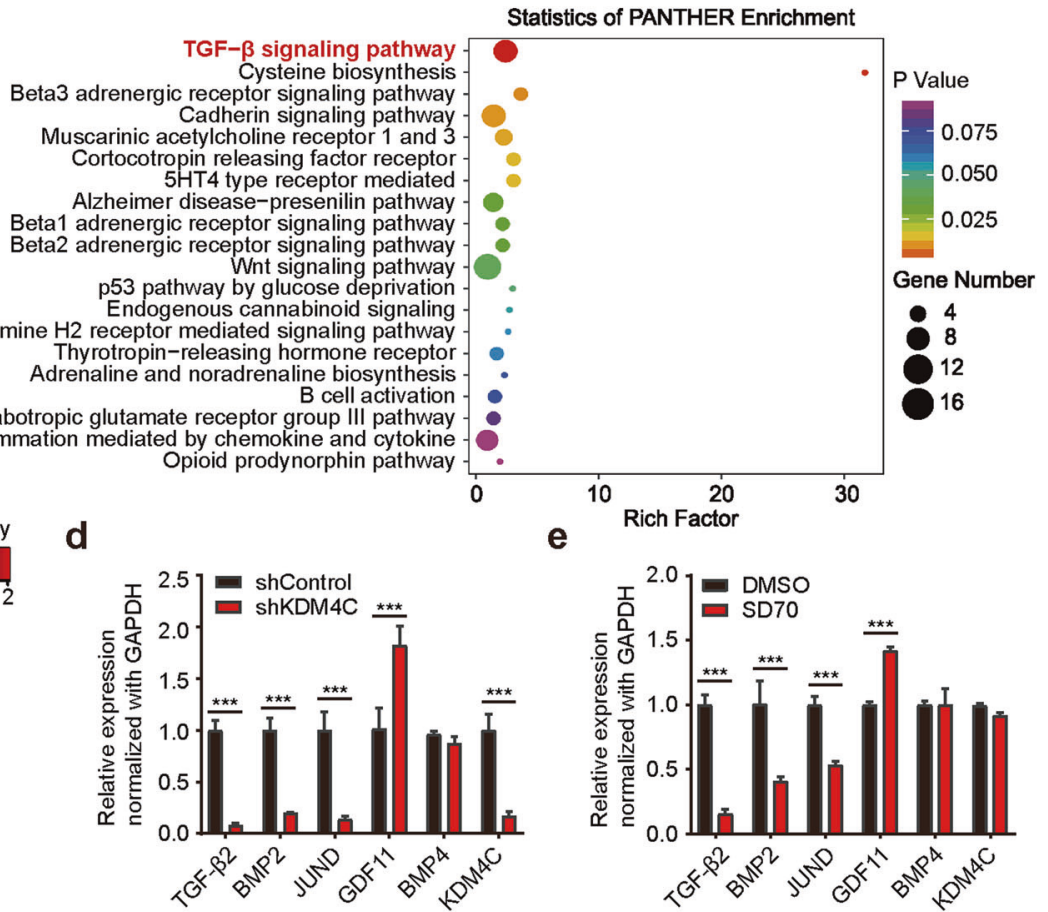

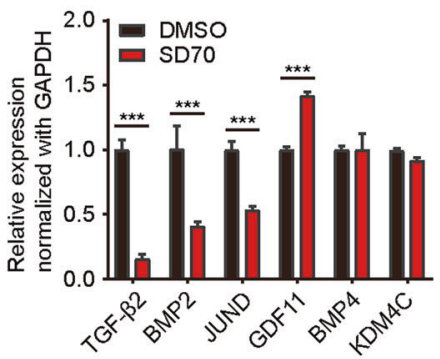

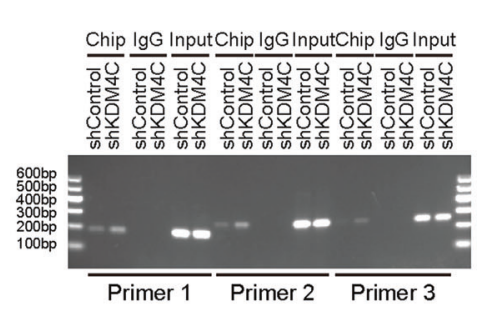
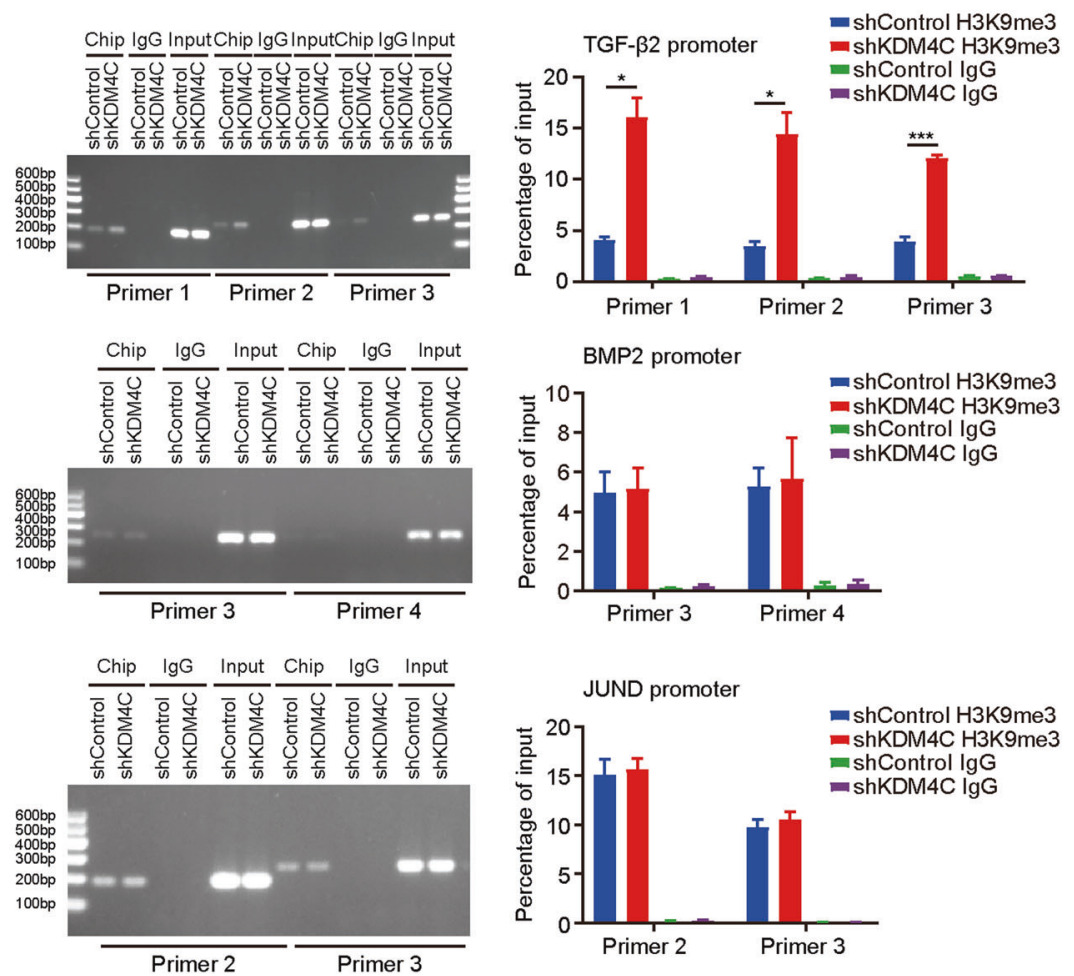

Fig. 4 KDM4C prevents H3K9me3 accumulation at the TGF- $\beta 2$ promoter to activate the TGF- $\beta$ /Smad signaling pathway. a Volcano plot comparing control and KDM4C knockdown SPC-A1 cells. b Enrichment of differentially expressed genes in signaling pathways is illustrated in an advanced bubble chart. $Y$-axis represents pathways, and the $X$-axis represents rich factor. Size and color of the bubble are representation of the amount of differentially expressed genes enriched in pathways and their enrichment significance, respectively. c Heat map produced from RNA sequencing. d, e mRNA levels of indicated

genes were measured using quantitative real-time PCR. $* * * P<0.001$ $(n=3)$. f-h An H3K9me3 ChIP assay was performed in shKDM4C and shControl cells. Left panel: ChIP primers were designed spanning from -2000 to $+200 \mathrm{bp}$ around the transcription start sites of indicated genes. Middle panel: Representative images of gel electrophoresis. Right panel: The H3K9me3 level in the gene promoter region was quantified and normalized to the input. IgG was used as the negative control. $* P<0.05 ; * * P<0.01 ; * * * P<0.001(n=3)$. 


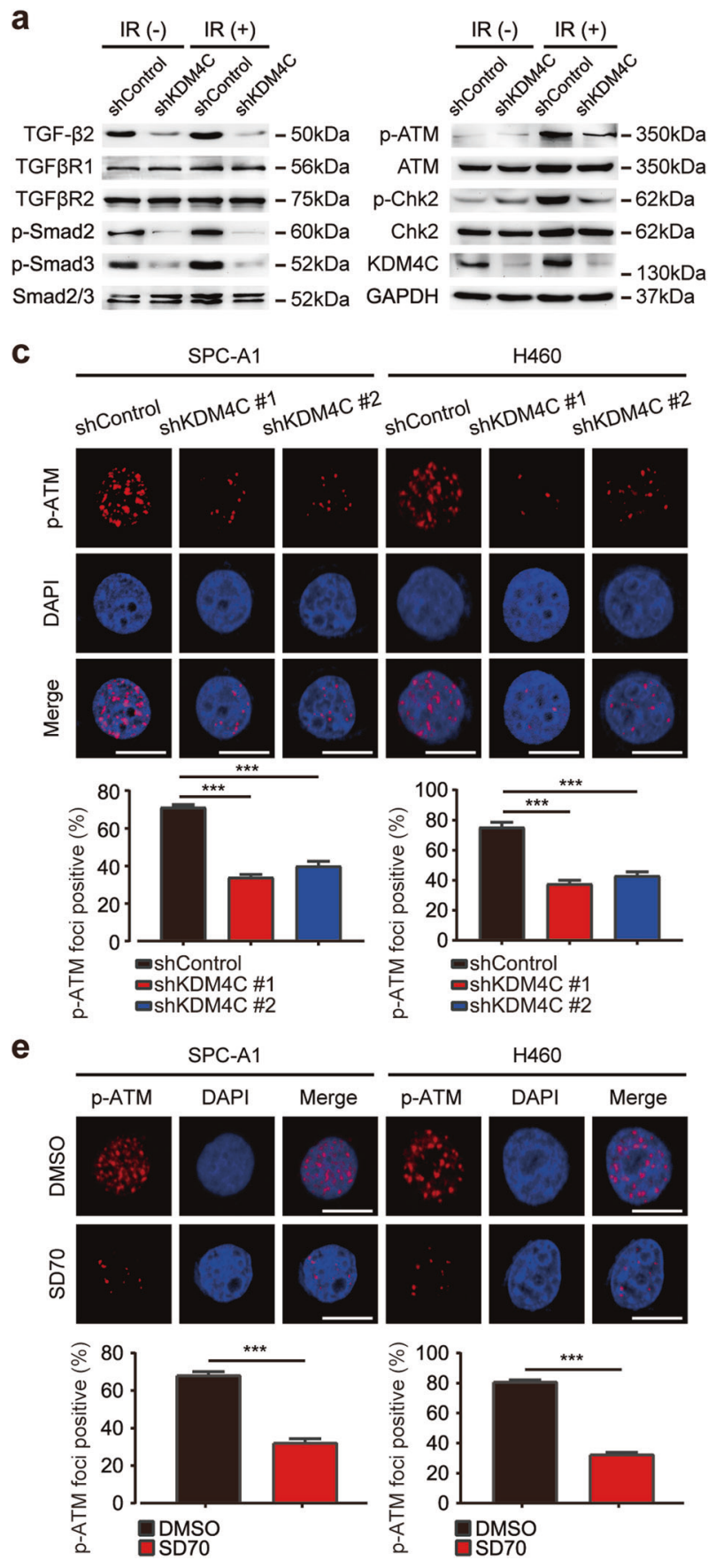

Fig. 5 KDM4C is a key activator of the TGF-B2/Smad/ATM signaling pathway. $\mathbf{a}, \mathbf{b}$ Changes in essential components of the TGF- $\beta$ signaling pathway after genetical or pharmacological inhibition of KDM4C in SPC-A1 cells $(n=3)$. c, $\mathbf{d}$ SPC-A1 cells stably expressing control or KDM4C shRNAs were exposed to irradiation and collected $4 \mathrm{~h}$ later. Immunostaining was performed to examine p-ATM and

More importantly, the addition of recombinant human TGF- $\beta 2$ partially rescued the declined levels of p-Smad2 and p-Smad3 in KDM4C-depleted cells (Fig. 6a). Functional experiments demonstrated that exogenous TGF- $\beta 2$ partially reversed the inhibition of cell growth and the increase of radiosensitivity b

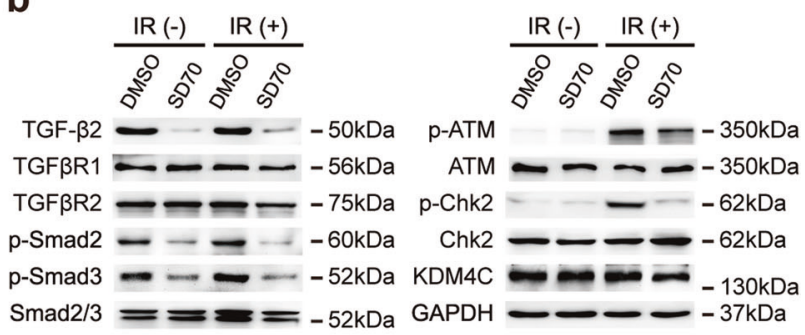

d

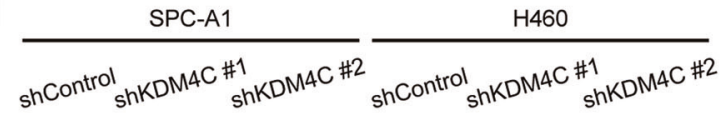
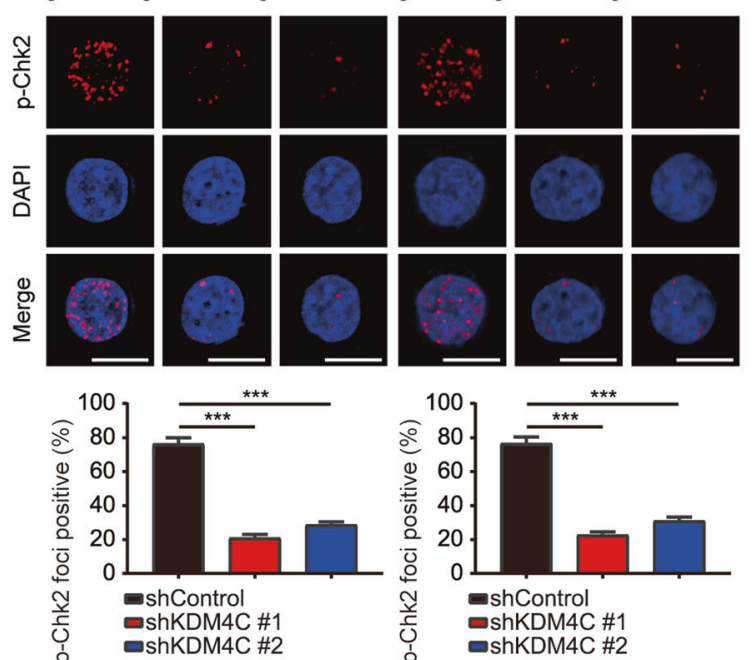

f

f
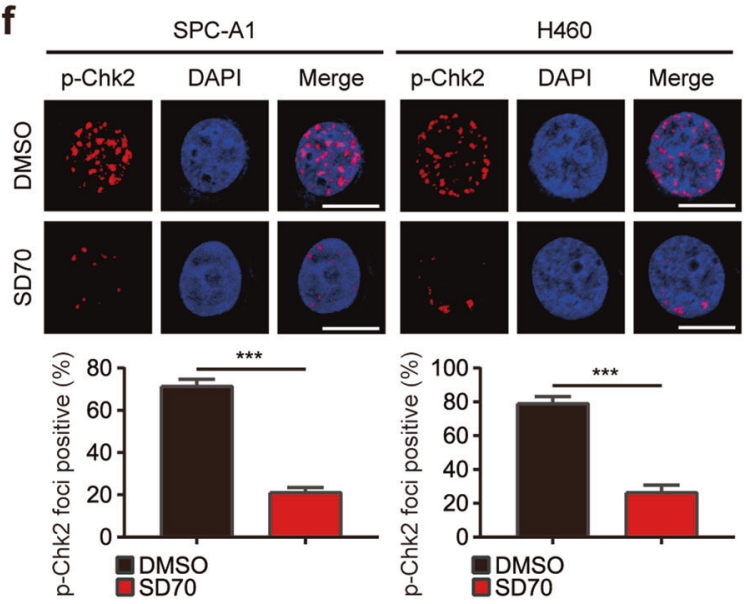

p-Chk2 foci formation. $* * * P<0.001 \quad(n=3)$. Scale bar, $10 \mu \mathrm{m}$. e, f SPC-A1 cells were treated with SD70 $(3 \mu \mathrm{M})$ for $24 \mathrm{~h}$ and $48 \mathrm{~h}$, respectively prior to exposure to irradiation. Cells were harvested after $4 \mathrm{~h}$ and subjected to immunostaining using p-ATM and p-Chk2 antibodies. Foci formation was quantified under a fluorescent microscope. $* * * P<0.001(n=3)$. Scale bar, $10 \mu \mathrm{m}$.

caused by KDM4C knockdown (Fig. 6c, e, g and i). These data reveal that $\mathrm{KDM} 4 \mathrm{C}$ promotes lung cancer cell proliferation and radioresistance in a TGF- $\beta 2 /$ Smad-dependent manner. To further investigate whether the TGF- $\beta 2 / \mathrm{Smad}$ pathway is required for the biological functions of KDM4C in 
a
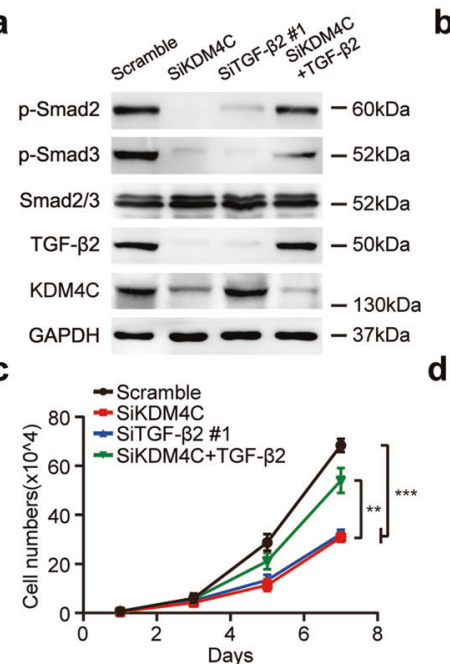

b

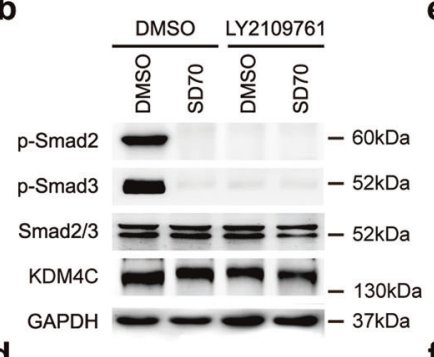

d

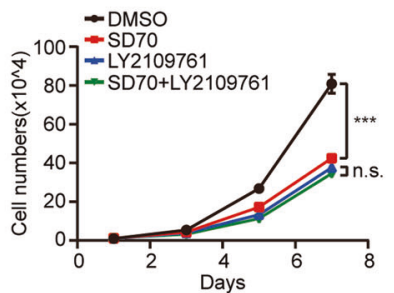

e



f

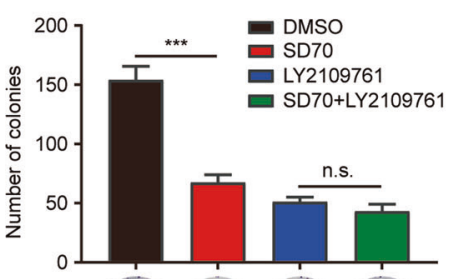

g

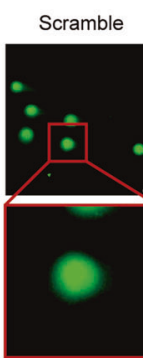

SiKDM4C
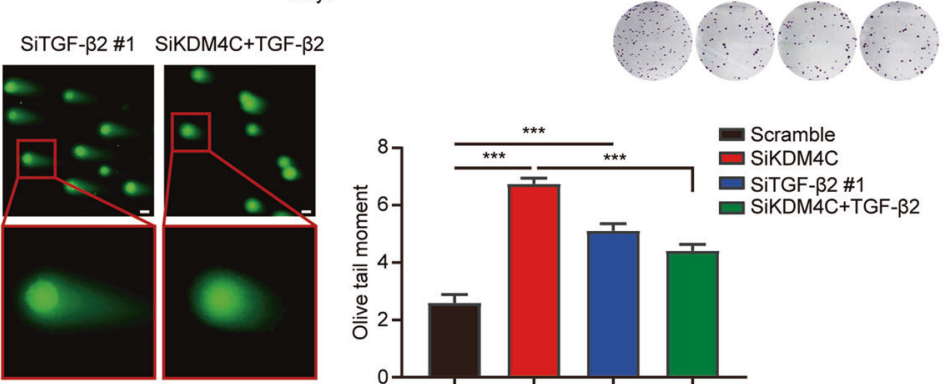

h

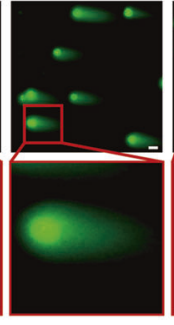

LY2109761 SD70+LY2109761
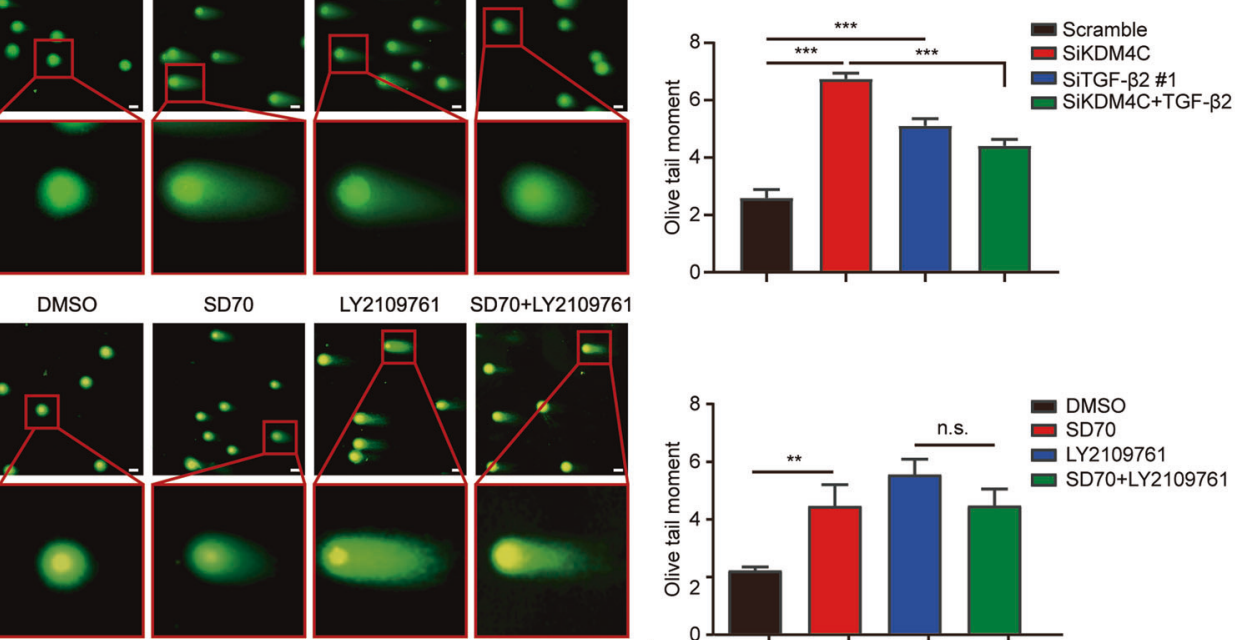

SD70
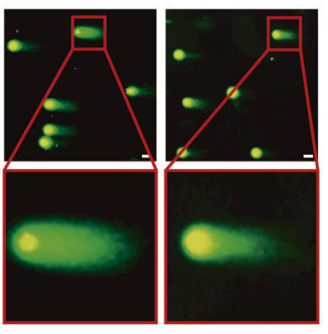

i
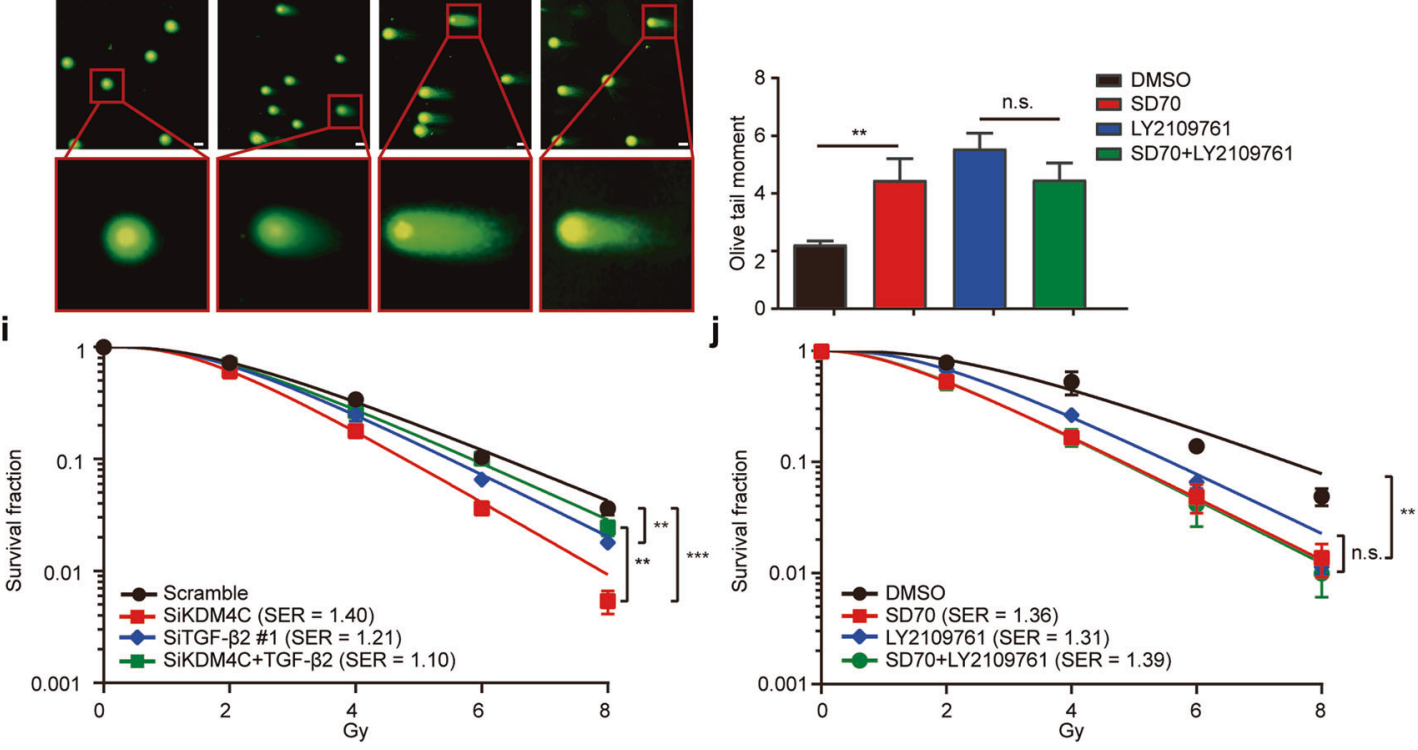

Fig. 6 The TGF-ק2/Smad signaling pathway activation is required for KDM4C-mediated effects on tumor growth and radioresistance in lung cancer. a SPC-A1 cells transfected with indicated siRNAs in the absence or presence of $4 \mathrm{ng} / \mathrm{ml}$ TGF- $\beta 2$ for $24 \mathrm{~h}$ were collected and analyzed by Western blotting using indicated antibodies $(n=3)$. b SPC-A1 cells treated with LY2109761 $(10 \mu \mathrm{M})$, SD70 (3 $\mu \mathrm{M})$ or a combination of both were collected and analyzed by immunoblotting $(n=3)$. c, d SPC-A1 cells treated with indicated siRNAs or drugs were seeded at low density in 6-well plates. Cell numbers were counted every other day. ${ }^{* *} P<0.01, * * * P<0.001$

$(n=3)$. e, f SPC-A1 cells treated with indicated siRNAs or drugs were plated in triplicates. Histograms representing the numbers of colonies are depicted. $* * * P<0.001(n=3)$. n. s. indicates no statistically significant difference $(P>0.05, n=3)$. g, h Neutral comet assay performed after exposure to irradiation. Left panel: Representative images show comet tail. Scale bar, $10 \mu \mathrm{m}$. Right panel: the olive tail moment was quantified and graphed for each group. $* * P<0.01$, $* * * P<0.001$ $(n=3)$. i, $\mathbf{j}$ SPC-A1 cells treated with indicated siRNAs or drugs were irradiated using indicated doses. Colonies containing more than 50 cells were recorded after 2 weeks. $* * P<0.01, * * * P<0.001(n=3)$. 
a

\begin{tabular}{cc}
\multicolumn{2}{c}{ KDM4C (bait) } \\
\hline Protein & Number of Peptides \\
\hline KDM4C & 78 \\
VIM & 7 \\
USP9X & 4 \\
PPP2R2A & 4 \\
IRS4 & 4 \\
FBXO22 & 4 \\
RNH1 & 3 \\
EEF2 & 3 \\
UBA1 & 2 \\
RPL3 & 2 \\
\hline
\end{tabular}

d
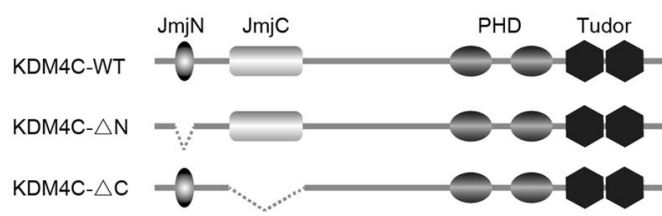

KDM $4 \mathrm{C}-\triangle \mathrm{P}$

$K D M 4 C-\triangle T$

f

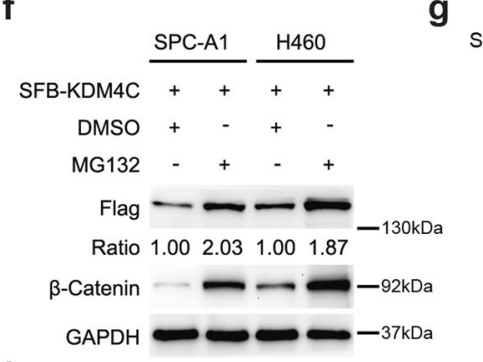

i

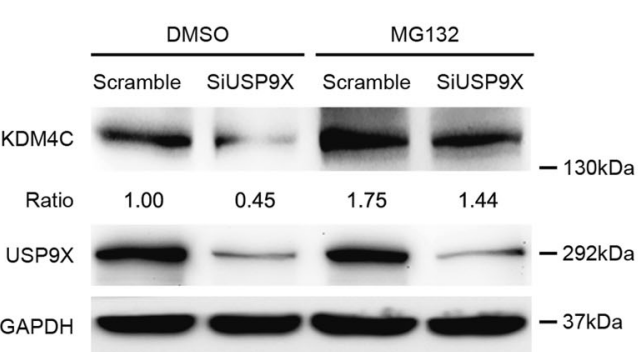

$\mathbf{k}$

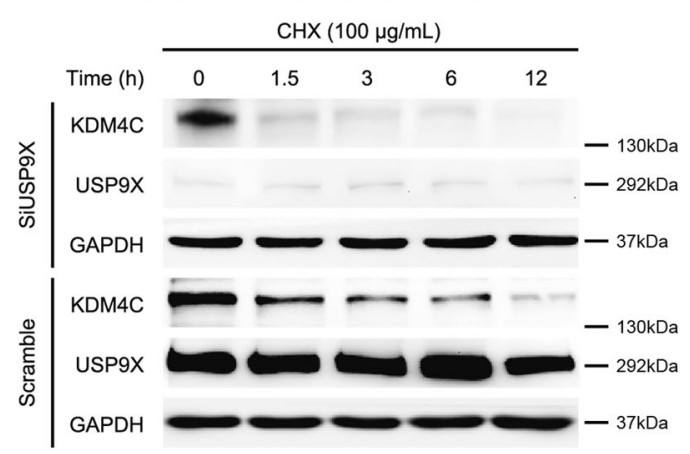

lung cancer, cells blocked pharmacologically using SD70 were simultaneously treated with LY2109761, a novel TGF- $\beta$ receptor type I/II dual inhibitor which inhibits the activation of the TGF- $32 /$ Smad signaling pathway (Fig. 6b). As shown in Fig. 6d, f, h and j, the addition of LY2109761 inhibited proliferation and resulted in increased olive tail moment and sensitivity to IR, which is consistent with its reported actions.

\section{C}

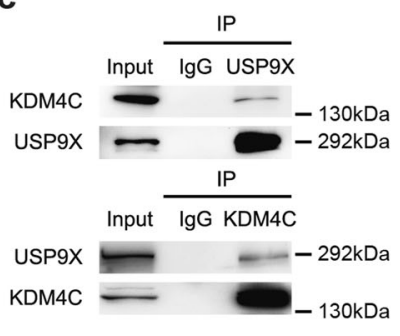

\section{e}

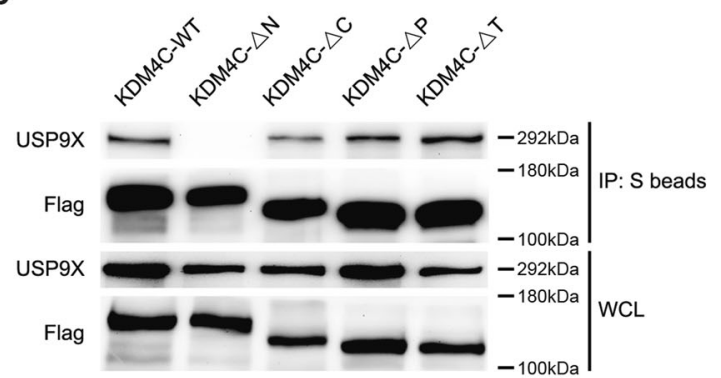

h

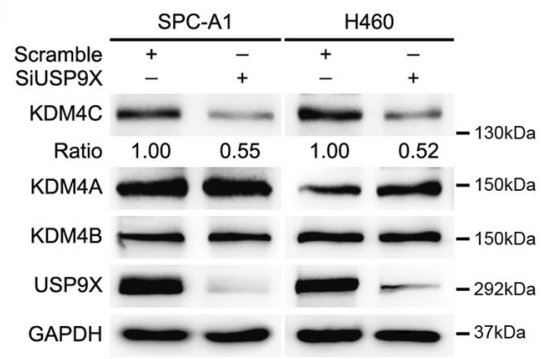

j
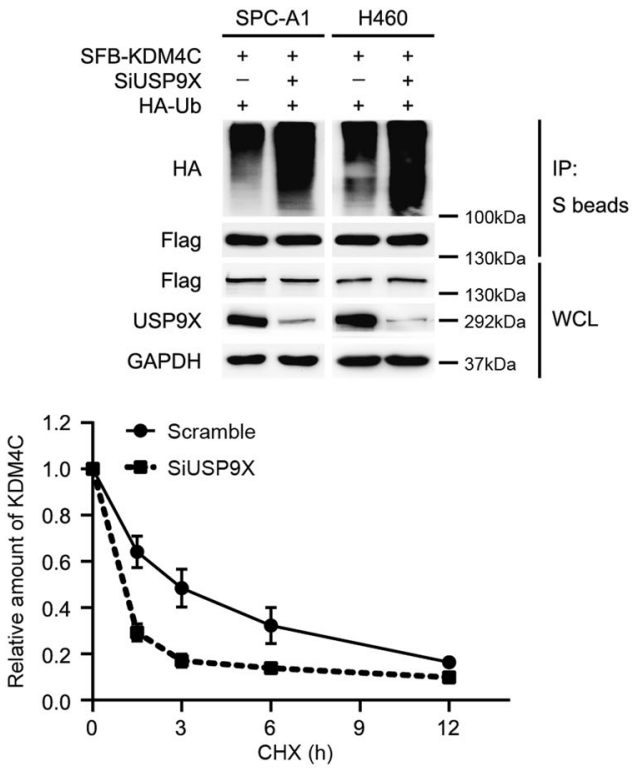

Strikingly, we found that further inhibition of KDM4C using pharmacological blockade has minimal effect on cell growth and radiosensitivity when cells were simultaneously treated with LY2109761 (Fig. $6 \mathrm{~d}, \mathrm{f}, \mathrm{h}$ and j). Together, our results indicate that $\mathrm{KDM} 4 \mathrm{C}$ exerts its biological functions mainly via its ability to activate the TGF- $\beta 2 / \mathrm{Smad}$ signaling pathway. 
Fig. 7 The deubiquitinase USP9X binds to the JmjN domain of KDM4C to prevent its degradation. a TAP-MS analysis shows KDM4C interacting proteins in HEK293T cells. The name and number of peptides for each protein identified are listed. b SPC-A1 and H460 cells were transfected with SFB-KDM4C plasmids. $24 \mathrm{~h}$ later, cells were harvested and then incubated with S-protein agarose prior to analysis by Western blotting $(n=3)$. c Endogenous interaction between KDM4C and USP9X in SPC-A1 cells $(n=3)$. d Schematic description of the domains of $\mathrm{KDM} 4 \mathrm{C}$ and the generated deletion mutants. e The JmjN domain of KDM4C is required for its binding to USP9X. S protein beads were used for immunoprecipitation assay and cell lysates were subjected to immunoblotting $(n=3)$. WCL: whole cell lysate. f SPC-A1 and H460 cells were transfected with a plasmid encoding KDM4C for $24 \mathrm{~h}$ and then incubated with MG132 $(10 \mu \mathrm{M})$ for $4 \mathrm{~h}$ prior to harvesting $(n=3)$. $\mathrm{g}$ SPC-A1 cells were transfected with the indicated constructs for $24 \mathrm{~h}$, and MG132 $(10 \mu \mathrm{M})$ was added for another $4 \mathrm{~h}$. Cell lysates were then subjected to immunoprecipitation using S-protein beads and subsequent analysis by Western blotting $(n=3)$. h USP9X knockdown results in reduced levels of endogenous $\mathrm{KDM} 4 \mathrm{C}(n=3)$. i SPC-A1 cells were transfected with the indicated siRNAs for $48 \mathrm{~h}$, incubated with DMSO or $10 \mu \mathrm{M}$ MG132 for another $4 \mathrm{~h}$. Cells were collected and probed for the indicated proteins by Western blotting $(n=3)$. $\mathbf{j}$ Cells were transfected with the indicated siRNAs and plasmids prior to treatment with MG132 (10 $\mu \mathrm{M})$ for $4 \mathrm{~h}$ before collection. The lysates were incubated with $\mathrm{S}$ beads overnight and then subjected to immunoblotting $(n=3)$. k Left panel: SPC-A1 cells transfected with the indicated siRNAs for $48 \mathrm{~h}$ were treated with $100 \mu \mathrm{g} / \mathrm{mL}$ of cycloheximide (CHX) and collected at the indicated time points. Immunoblotting was performed to examine the protein level of KDM4C. Right panel: quantification of the KDM4C band intensity $(n=3)$.

\section{The deubiquitinase USP9X binds to the JmjN domain of KDM4C to prevent its degradation}

To explore how KDM4C is upregulated in lung cancer, unbiased tandem affinity purification (TAP) and mass spectrometry (MS) proteomic analysis were conducted to identify KDM4C-interacting proteins. Excitingly, the deubiquitinase USP9X was one of the potential KDM4C binding proteins (Fig. 7a). To confirm this result, we carried out coimmunoprecipitation assays and found that exogenously transfected SFB-tagged KDM4C was able to form a complex with USP9X (Fig. 7b). In addition, we also showed the presence of an endogenous interaction between USP9X and KDM4C in lung cancer cells (Fig. 7c). Next, to map the domains of KDM4C responsible for binding to USP9X, we constructed a series of KDM4C deletion mutants and found that the JmjN domain deletion mutant failed to form a complex with USP9X, indicating that the JmjN domain of KDM4C binds to USP9X (Fig. 7d, e). Collectively, these results confirm that USP9X physically and specifically interacts with KDM4C in lung cancer cells.

USP9X is a deubiquitinase that has the ability to stabilize the ubiquitination substrate [21-23], therefore, we speculated that USP9X might affect the stability of KDM4C via the ubiquitin/proteasome pathway. Indeed, we observed marked protein accumulation and increased polyubiquitination of $\mathrm{KDM} 4 \mathrm{C}$ after treatment with the proteasome inhibitor MG132 (Fig. 7f, g), indicating that KDM4C can be degraded by the proteasome. Moreover, depletion of USP9X with specific siRNAs, which have been described by other groups [21-23], resulted in a downregulation of $\mathrm{KDM} 4 \mathrm{C}$, while KDM4A and KDM4B remained unchanged (Fig. 7h). More importantly, we found that MG132 treatment substantially rescued the decline in KDM4C protein caused by USP9X depletion in lung cancer cells (Fig. 7i), implying that USP9X protects KDM4C from degradation by the proteasome. Furthermore, we demonstrated that USP9X silencing increased the polyubiquitination and shortened the half-life of KDM4C (Fig. 7j, k). These data provide substantial evidence that USP9X stabilizes KDM4C by preventing its degradation.

\section{USP9X silencing impairs TGF- $\beta / S m a d$ signaling and radioresistance by destabilizing KDM4C in lung cancer cells}

To further clarify the role of USP9X in lung cancer radioresistance and confirm whether this effect is dependent on KDM4C, we transfected exogenously expressed KDM4C into USP9X-depleted cells and found that TGF- $\beta 2$, pSmad2 and p-Smad3 protein levels were decreased when USP9X was knocked down (Fig. 8a). In addition, downregulation of USP9X also led to reduced levels of TGF- $\beta 2$ mRNA in lung cancer cells (Fig. 8b). Importantly, restoration of KDM4C partially rescued the reduced levels of TGF- $\beta 2$, p-Smad2 and p-Smad3 in USP9X-depleted lung cancer cells (Fig. 8a, b), suggesting that USP9X silencing inhibits TGF- $\beta 2 /$ Smad signaling in a KDM4C-dependent manner. We further observed that loss of USP9X suppressed the growth and proliferation of lung cancer cells, and these defects were partially restored by overexpression of KDM4C (Fig. 8c, d). In addition, our data also showed that USP9X-depleted lung cancer cells exhibited increased olive tail moment and enhanced radiosensitivity, and these phenotypes were also partially rescued by reexpressing KDM4C (Fig. 8e, f). Together, these results indicate that USP9X activates TGF- $\beta 2 /$ Smad signaling and promotes radioresistance by stabilizing KDM4C in lung cancer cells.

\section{Discussion}

In this study, we provide substantial evidence that supports the novel role of $\mathrm{KDM} 4 \mathrm{C}$ in promoting radioresistance in lung cancer. Targeting of KDM4C with genetical or pharmacological inhibition significantly overcomes lung cancer radioresistance in vitro and in vivo. Furthermore, we provide new insights into the potency and efficacy of the 
a

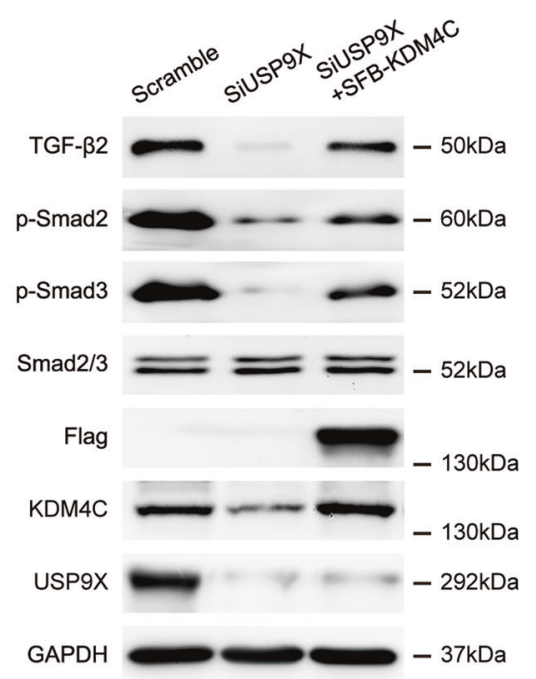

e

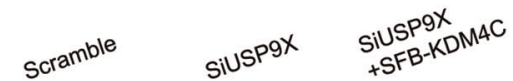

b

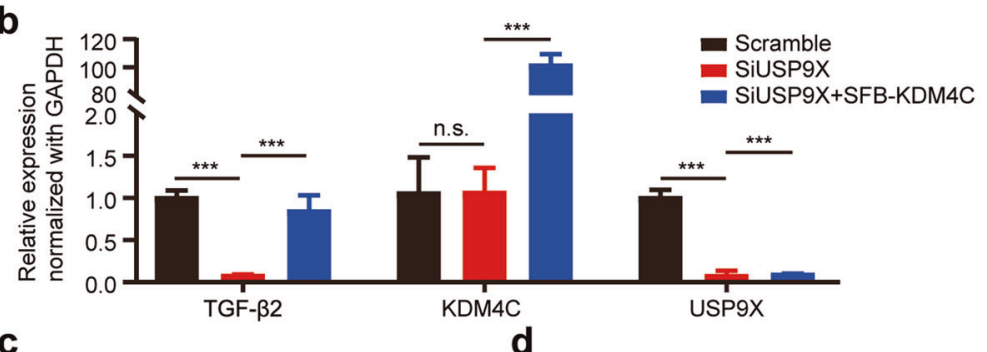

C
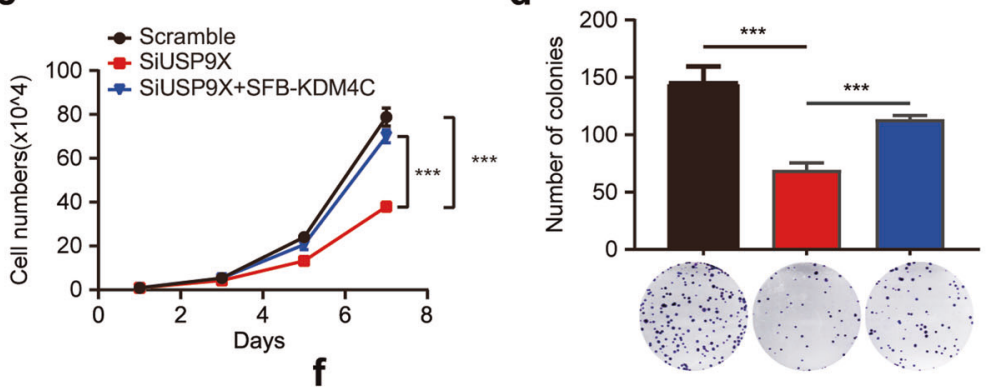
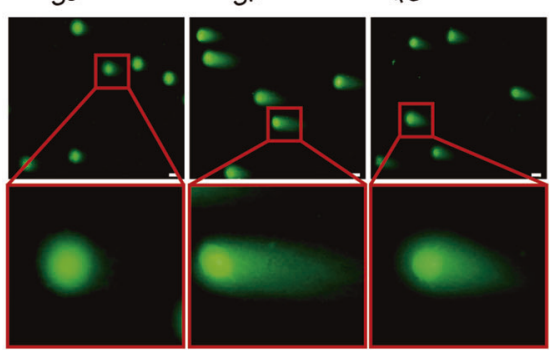

- Scramble

- SiUSP9X

- SiUSP9X+SFB-KDM4C
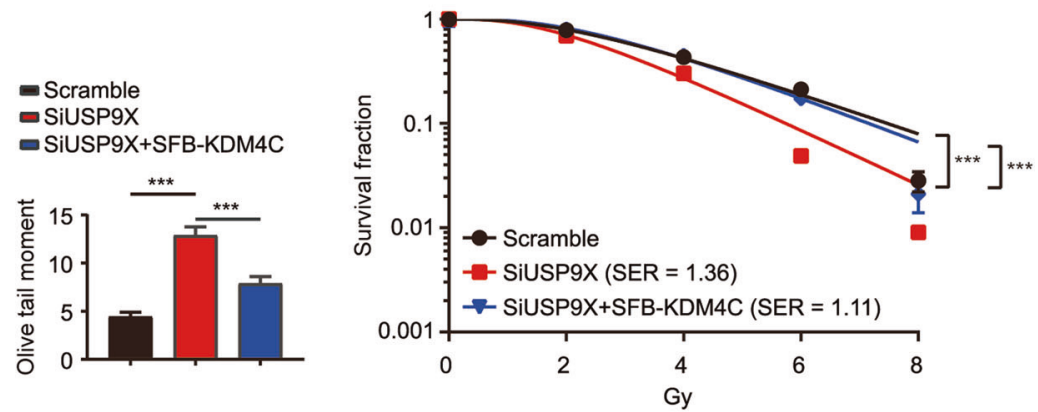

g

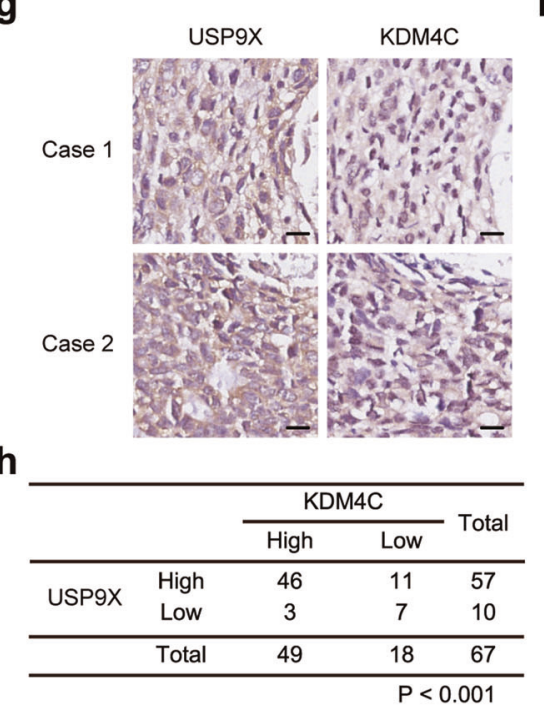

i

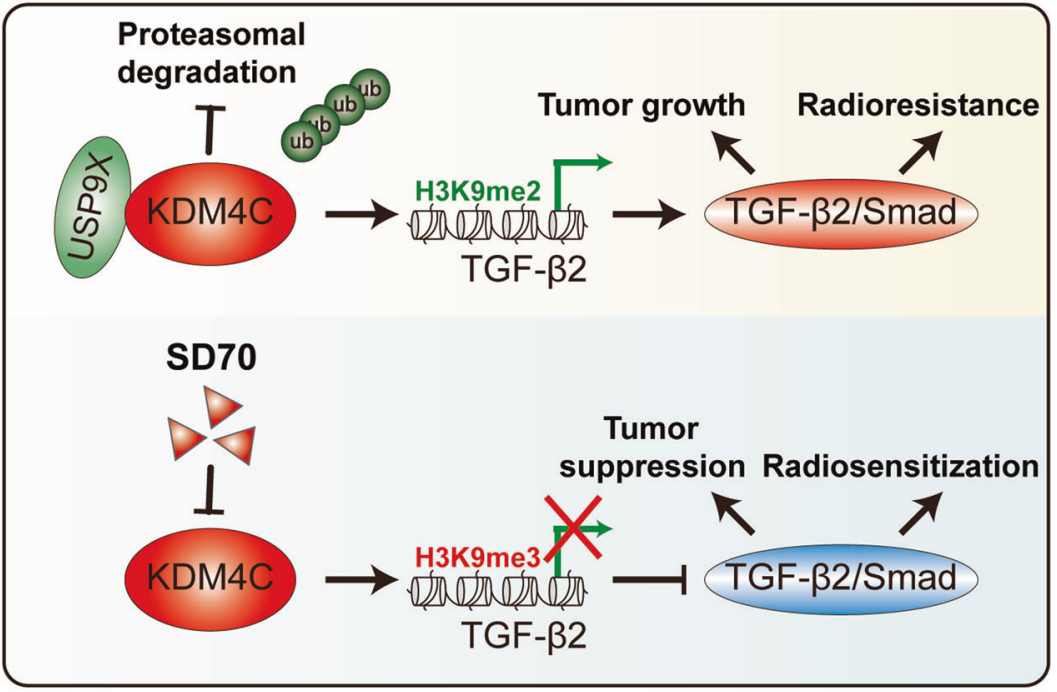

epigenetic drug SD70 as a radiosensitizer in the treatment of lung cancer. Importantly, we identify the deubiquitinase USP9X as a novel upstream regulator which interacts with and stabilizes KDM4C and reveal that KDM4C-mediated H3K9me3 removal at the TGF- $\beta 2$ promoter activates TGF$\beta 2 / \mathrm{Smad} / \mathrm{ATM}$ signaling. To our knowledge, this is the first report uncovering the implication of $\mathrm{KDM} 4 \mathrm{C}$ in the regulation of lung cancer radioresistance.
The ubiquitin-proteasome system is the major regulator of intracellular protein degradation and turnover, and dysregulation of this system can result in overexpression of oncoproteins or downregulation of tumor suppressors, eventually leading to tumorigenesis [33]. Notably, the ubiquitination modification is a dynamic and reversible process since deubiquitinating enzymes (DUBs) are responsible for the specific removal of ubiquitin from proteolysis-targeted 
Fig. 8 USP9X promotes tumor progression and radioresistance by stabilizing KDM4C in lung cancer. a SPC-A1 cells transfected with indicated siRNAs or SFB-KDM4C plasmid were collected and analyzed by Western blotting using indicated antibodies $(n=3)$. b SPCA1 cells were transfected with indicated siRNAs or SFB-KDM4C plasmid, followed by real-time PCR to determine the mRNA levels of TGF- $\beta 2$, KDM4C and USP9X. ${ }^{* * *} P<0.001(n=3)$. c SPC-A1 cells transfected with the indicated siRNAs and plasmids were seeded and calculated every other day. Data are shown as the mean $\pm \mathrm{SD}$. $* * * P<$ $0.001(n=3)$. d SPC-A1 cells transfected with the indicated siRNAs and plasmids were seeded and cultured for 2 weeks. The colonies were stained using crystal violet solution and then counted under a microscope. $* * * P<0.001 \quad(n=3)$. e Representative pictures of Comet assays performed $4 \mathrm{~h}$ after IR. $* * * P<0.001(n=3)$. Scale bar, $20 \mu \mathrm{m}$. f SPC-A1 cells transfected with the indicated siRNAs and plasmids were irradiated with indicated doses. The percentages of surviving colonies were evaluated 2 weeks later. $* * * P<0.001 \quad(n=3)$. g Representative immunohistochemical staining for USP9X and KDM4C in lung cancer tissues. Scale bar, $10 \mu \mathrm{m}$. h Positive correlation between USP9X and KDM4C protein levels in lung cancer tissues $(P<0.001$, chi-square test). i Schematic diagram summarizes aberrant epigenetic networks and therapeutic potentials of targeting KDM4C in lung cancer. Upper panel: Deubiquitinase USP9X-mediated aberrant recruitment of KDM4C activates TGF- $\beta 2 / \mathrm{Smad}$ signaling to promote lung cancer progression and radioresistance. Lower panel: Pharmacological targeting of KDM4C using SD70 inhibits the activation of TGF- $\beta 2 /$ Smad signaling, which in turn leads to enhanced radiosensitization.

proteins and contribute to its stability [33]. Available evidence shows that KDM4C may act as an oncoprotein, however, the underlying upstream regulatory mechanisms remain elusive. We are first to uncover that deubiquitinase USP9X is a novel specific upstream modulator that controls the stability of KDM4C. USP9X, also called FAM, is frequently dysregulated in multiple cancers and plays an oncogenic role in lung cancer progression [22, 34-36]. In our study, we found that USP9X is highly expressed and correlated with poor overall survival in lung cancer patients (Supplementary Fig. 4). Moreover, we also uncovered that USP9X preferentially upregulates the protein levels of $\mathrm{KDM} 4 \mathrm{C}$ by preventing its ubiquitination in lung cancer. This is the first report to reveal that KDM4C can be regulated and deubiquitinated at the posttranslational level. In addition, we revealed that USP9X is involved in lung cancer progression and radioresistance in a KDM4Cdependent manner. Given that USP9X co-localized with KDM4C and a significant positive correlation between the expression of USP9X and KDM4C in lung cancer tissues was observed (Fig. 8g, h and Supplementary Fig. 5), we conclude that USP9X overexpression may account for KDM4C upregulation in human lung cancer. Thus, our results strongly support a model that KDM4C is a novel substrate of deubiquitinase USP9X and its overproduction is the result of the deubiquitination modification by USP9X.

Aberrant expression of KDM4C has been observed in several types of cancers [5, 6], indicating its oncogenic roles in tumorigenesis. Our clinical data show that KDM4C is overexpressed and associated with poor prognosis in lung cancer. In addition, the functional results reveal that genetic deletion or pharmacological inhibition of KDM4C suppressed tumor growth in vitro and in vivo, further supporting our hypothesis that $\mathrm{KDM} 4 \mathrm{C}$ acts as an oncoprotein in lung cancer. More importantly, we uncover a novel role of $\mathrm{KDM} 4 \mathrm{C}$ in promoting lung cancer radioresistance. Our results show that KDM4C inhibition brought about increased DNA damage and subdued DNA repair response, rendering lung cancer cells and xenograft tumors more susceptible to IR. Mechanistically, we reveal that KDM4C activates TGF- $\beta 2 /$ Smad signaling by promoting the removal of H3K9me 3 at the TGF- $\beta 2$ promoter. KDM4C inhibition reduces the expression of TGF- $\beta 2$ and decreased the protein levels of p-Smad 2 and p-Smad 3 in lung cancer cells. In addition, our findings show that KDM4C activates TGF- $\beta 2 /$ Smad signaling and its downstream ATM/Chk2 pathway, which may explain the mechanism behind increased radiosensitivity after KDM4C inhibition since Smad2 is a critical intermediary between the TGF- $\beta$ and ATM signaling crosstalk essential in DNA repair [16, 17]. Therefore, these data support a notion that USP9X-mediated deubiquitination of KDM4C stimulates the activation of the TGF$\beta 2 / \mathrm{Smad} / \mathrm{ATM}$ signaling, and eventually contributes to radioresistance in lung cancer.

Epigenetic drugs such as histone deacetylase inhibitors and DNA-methyltransferase inhibitors have been widely used in lymphohematopoietic cancer therapy [37]. SD70, a selective inhibitor of KDM4C, is a newly developed epigenetic drug. We for the first time uncovered the roles of SD70 as an anti-cancer agent and radiosensitizer in lung cancer. Inhibition of KDM4C with SD70 can not only suppress cell growth in vitro and in vivo but also accelerate DNA damage and inhibit DNA repair, ultimately enhancing radiosensitivity in lung cancer. In our xenograft mouse model, combined treatment with relatively low SD70 concentration and IR exhibited a strong synergistic inhibitory effect on lung cancer. Thus, our findings provide a rationale for future clinical trials of combined treatment with SD70 and radiotherapy in lung cancer patients.

In summary, our study identifies the upstream modulator and downstream mediator of KDM4C and reveals that targeting KDM4C via blocking TGF- $\beta 2 / S m a d$ signaling impairs tumorigenesis and enhances radiosensitivity in lung cancer (Fig. 8i). More importantly, we provide preclinical evidence of the potency of SD70 as an attractive anti-cancer agent and radiosensitizer that may bring about prolonged overall survival in lung cancer patients.

Funding This work was supported by the National Natural Science Foundation of China (81874218 and 81874041); the Hubei Provincial Natural Science Fund for Distinguished Young Scholars (2019CFA063) and the grants from the Wuhan Science and Technology Bureau (2019020701011437 and 2018060401011318). 
Author contributions SX conceived and designed the study, SX and GW supervised the study, XJ, WPF and RZ performed experiments and analyzed the data, YZ, YZ, RM, SZ, XD, TZ and KY provided advice and technical assistance, SX wrote the paper. All authors have contributed to and approved the final paper.

\section{Compliance with ethical standards}

Conflict of interest The authors declare that they have no conflict of interest.

Ethical approval All animal experiments were approved by the Medical Ethics Committee of Tongji Medical College, Huazhong University of Science and Technology.

Publisher's note Springer Nature remains neutral with regard to jurisdictional claims in published maps and institutional affiliations.

Open Access This article is licensed under a Creative Commons Attribution 4.0 International License, which permits use, sharing, adaptation, distribution and reproduction in any medium or format, as long as you give appropriate credit to the original author(s) and the source, provide a link to the Creative Commons license, and indicate if changes were made. The images or other third party material in this article are included in the article's Creative Commons license, unless indicated otherwise in a credit line to the material. If material is not included in the article's Creative Commons license and your intended use is not permitted by statutory regulation or exceeds the permitted use, you will need to obtain permission directly from the copyright holder. To view a copy of this license, visit http://creativecommons. org/licenses/by/4.0/.

\section{References}

1. Flavahan WA, Gaskell E, Bernstein BE. Epigenetic plasticity and the hallmarks of cancer. Science. 2017;357:eaal2380.

2. Berry WL, Janknecht R. KDM4/JMJD2 histone demethylases: epigenetic regulators in cancer cells. Cancer Res. 2013;73:2936-42.

3. Cloos PA, Christensen J, Agger K, Maiolica A, Rappsilber J, Antal T, et al. The putative oncogene GASC1 demethylates triand dimethylated lysine 9 on histone H3. Nature. 2006;442:307-11.

4. Yang ZQ, Imoto I, Fukuda Y, Pimkhaokham A, Shimada Y, Imamura M, et al. Identification of a novel gene, GASC1, within an amplicon at 9p23-24 frequently detected in esophageal cancer cell lines. Cancer Res. 2000;60:4735-9.

5. Luo W, Chang R, Zhong J, Pandey A, Semenza GL. Histone demethylase JMJD2C is a coactivator for hypoxia-inducible factor 1 that is required for breast cancer progression. Proc Natl Acad Sci USA. 2012;109:E3367-3376.

6. Crea F, Sun L, Mai A, Chiang YT, Farrar WL, Danesi R, et al. The emerging role of histone lysine demethylases in prostate cancer. Mol Cancer. 2012;11:52.

7. Wissmann M, Yin N, Muller JM, Greschik H, Fodor BD, Jenuwein $\mathrm{T}$, et al. Cooperative demethylation by JMJD2C and LSD1 promotes androgen receptor-dependent gene expression. Nat Cell Biol. 2007;9:347-53.

8. Gregory BL, Cheung VG. Natural variation in the histone demethylase, KDM4C, influences expression levels of specific genes including those that affect cell growth. Genome Res. 2014;24:52-63.

9. Agger K, Miyagi S, Pedersen MT, Kooistra SM, Johansen JV, Helin K. Jmjd2/Kdm4 demethylases are required for expression of
Il3ra and survival of acute myeloid leukemia cells. Genes Dev. 2016;30:1278-88.

10. Jiang $\mathrm{Y}, \mathrm{Li} \mathrm{C}, \mathrm{Wu} \mathrm{Q}$, An $\mathrm{P}$, Huang $\mathrm{L}$, Wang $\mathrm{J}$, et al. Irondependent histone 3 lysine 9 demethylation controls B cell proliferation and humoral immune responses. Nat Commun. 2019;10:2935.

11. Jin C, Yang L, Xie M, Lin C, Merkurjev D, Yang JC, et al. Chemseq permits identification of genomic targets of drugs against androgen receptor regulation selected by functional phenotypic screens. Proc Natl Acad Sci USA. 2014;111:9235-40.

12. Cheung N, Fung TK, Zeisig BB, Holmes K, Rane JK, Mowen KA, et al. Targeting Aberrant Epigenetic Networks Mediated by PRMT1 and KDM4C in Acute Myeloid Leukemia. Cancer Cell. 2016;29:32-48.

13. Yu Y, Feng XH. TGF- $\beta$ signaling in cell fate control and cancer. Curr Opin Cell Biol. 2019;61:56-63.

14. Batlle E, Massague J. Transforming Growth Factor-beta Signaling in Immunity and Cancer. Immunity. 2019;50:924-40.

15. Dubrovska A, Kanamoto T, Lomnytska M, Heldin CH, Volodko $\mathrm{N}$, Souchelnytskyi S. TGF $\beta 1 / \mathrm{Smad} 3$ counteracts BRCA1dependent repair of DNA damage. Oncogene. 2005;24:2289-97.

16. Wang M, Saha J, Hada M, Anderson JA, Pluth JM, O'Neill P, et al. Novel Smad proteins localize to IR-induced double-strand breaks: interplay between TGF $\beta$ and ATM pathways. Nucleic Acids Res. 2013;41:933-42.

17. Barcellos-Hoff MH, Cucinotta FA. New tricks for an old fox: impact of TGF $\beta$ on the DNA damage response and genomic stability. Sci Signal. 2014;7:re5.

18. Zhang M, Kleber S, Rohrich M, Timke C, Han N, Tuettenberg J, et al. Blockade of TGF- $\beta$ signaling by the TGF $\beta$ R-I kinase inhibitor LY2109761 enhances radiation response and prolongs survival in glioblastoma. Cancer Res. 2011;71:7155-67.

19. Bouquet F, Pal A, Pilones KA, Demaria S, Hann B, Akhurst RJ, et al. TGF $\beta 1$ inhibition increases the radiosensitivity of breast cancer cells in vitro and promotes tumor control by radiation in vivo. Clin Cancer Res. 2011;17:6754-65.

20. Pedersen MT, Agger K, Laugesen A, Johansen JV, Cloos PA, Christensen $\mathrm{J}$, et al. The demethylase JMJD2C localizes to $\mathrm{H} 3 \mathrm{~K} 4 \mathrm{me} 3$-positive transcription start sites and is dispensable for embryonic development. Mol Cell Biol. 2014;34:1031-45.

21. Schwickart M, Huang X, Lill JR, Liu J, Ferrando R, French DM, et al. Deubiquitinase USP9X stabilizes MCL1 and promotes tumour cell survival. Nature. 2010;463:103-7.

22. Chen X, Yu C, Gao J, Zhu H, Cui B, Zhang T, et al. A novel USP9X substrate TTK contributes to tumorigenesis in non-smallcell lung cancer. Theranostics. 2018;8:2348-60.

23. McGarry E, Gaboriau D, Rainey MD, Restuccia U, Bachi A, Santocanale C. The Deubiquitinase USP9X Maintains DNA Replication Fork Stability and DNA Damage Checkpoint Responses by Regulating CLASPIN during S-Phase. Cancer Res. 2016;76:2384-93.

24. Xu WW, Huang ZH, Liao L, Zhang QH, Li JQ, Zheng CC, et al. Direct Targeting of CREB1 with Imperatorin Inhibits TGF $\beta 2$ ERK Signaling to Suppress Esophageal Cancer Metastasis. Adv Sci. 2020;7:2000925.

25. Ma J, Lu Y, Zhang S, Li Y, Huang J, Yin Z, et al. $\beta$-Trcp ubiquitin ligase and RSK2 kinase-mediated degradation of FOXN2 promotes tumorigenesis and radioresistance in lung cancer. Cell Death Differ. 2018;25:1473-85.

26. Xu S, Li X, Gong Z, Wang W, Li Y, Nair BC, et al. Proteomic analysis of the human cyclin-dependent kinase family reveals a novel CDK5 complex involved in cell growth and migration. Mol Cell Proteom. 2014;13:2986-3000.

27. Yu Y, Schleich K, Yue B, Ji S, Lohneis P, Kemper K, et al. Targeting the Senescence-Overriding Cooperative Activity of 
Structurally Unrelated H3K9 Demethylases in Melanoma. Cancer Cell. 2018;33:322-36.

28. Wang Q, Ma J, Lu Y, Zhang S, Huang J, Chen J, et al. CDK20 interacts with KEAP1 to activate NRF2 and promotes radiochemoresistance in lung cancer cells. Oncogene. 2017;36:5321-30

29. Huang Y, Hu K, Zhang S, Dong X, Yin Z, Meng R, et al. S6K1 phosphorylation-dependent degradation of Mxil by $\beta$-Trcp ubiquitin ligase promotes Myc activation and radioresistance in lung cancer. Theranostics. 2018;8:1286-1300.

30. Zeng Y, Liu Q, Wang Y, Tian C, Yang Q, Zhao Y, et al. CDK5 Activates Hippo Signaling to Confer Resistance to Radiation Therapy Via Upregulating TAZ in Lung Cancer. Int J Radiat Oncol Biol Phys. 2020;108:758-69.

31. Olcina MM, Foskolou IP, Anbalagan S, Senra JM, Pires IM, Jiang Y, et al. Replication stress and chromatin context link ATM activation to a role in DNA replication. Mol Cell. 2013;52:758-66.
32. Ayrapetov MK, Gursoy-Yuzugullu O, Xu C, Xu Y, Price BD. DNA double-strand breaks promote methylation of histone $\mathrm{H} 3$ on lysine 9 and transient formation of repressive chromatin. Proc Natl Acad Sci USA. 2014;111:9169-74.

33. Ciechanover A. The unravelling of the ubiquitin system. Nat Rev Mol Cell Biol. 2015;16:322-4.

34. Li X, Song N, Liu L, Liu X, Ding X, Song X, et al. USP9X regulates centrosome duplication and promotes breast carcinogenesis. Nat Commun. 2017;8:14866.

35. Wang T, Jing B, Sun B, Liao Y, Song H, Xu D, et al. Stabilization of PTGES by deubiquitinase USP9X promotes metastatic features of lung cancer via PGE2 signaling. Am J Cancer Res. 2019;9:1145-60.

36. Chen Z, Wang HW, Wang S, Fan L, Feng S, Cai X, et al. USP9X deubiquitinates ALDH1A3 and maintains mesenchymal identity in glioblastoma stem cells. J Clin Investig. 2019;129:2043-55.

37. Berdasco M, Esteller M. Clinical epigenetics: seizing opportunities for translation. Nat Rev Genet. 2019;20:109-27. 